hep-ph/0404042

\title{
Toward precision measurements in solar neutrinos
}

\author{
P. C. de Holanda ${ }^{a}$, Wei Liao ${ }^{b}$ and A. Yu. Smirnov ${ }^{b, c}$ \\ ${ }^{a}$ Instituto de Fúsica Gleb Wataghin - UNICAMP, 13083-970 Campinas SP, Brasil \\ ${ }^{b}$ ICTP, Strada Costiera 11, 34014 Trieste, Italy \\ ${ }^{c}$ Institute for Nuclear Research of Russian Academy of Sciences, Moscow 117312, Russia
}

\begin{abstract}
Solar neutrino physics enters a stage of precision measurements. In this connection we present a precise analytic description of the neutrino conversion in the context of LMA MSW solution of the solar neutrino problem. Using the adiabatic perturbation theory we derive an analytic formula for the $\nu_{e}$ survival probability which takes into account the non-adiabatic corrections and the regeneration effect inside the Earth. The probability is averaged over the neutrino production region. We find that the non-adiabatic corrections are of the order $10^{-9}-10^{-7}$. Using the formula for the Earth regeneration effect we discuss features of the zenith angle dependence of the $\nu_{e}$ flux. In particular, we show that effects of small structures at the surface of the Earth can be important.
\end{abstract}

\section{Introduction}

The LMA MSW solution [1, 2] has been identified [3]-[15] as the correct solution of the solar neutrino problem. The $2 \nu$ conversion probability of this solution gives a very good description of all available data: no statistically significant deviation has been found so far. New physics effects beyond LMA, if exist, are below few per cent.

The program of future solar neutrino studies includes

1). further tests of the LMA solution, in particular, searches for signatures of this solution such as the Day-Night asymmetry and the distortion ("upturn") of the boron $\nu_{e}$ spectrum at low energies;

2). precise determination of the oscillation parameters, especially the 1-2 mixing angle;

3 ). searches for the sub-leading effects which originate from

- 1-3 mixing,

- sterile neutrino mixing,

- non-standard neutrino interactions,

- spin-flavor flip in the magnetic fields of the Sun,

- violation of the fundamental symmetries (CPT, equivalence principle, etc.).

Already the present solar neutrino measurements have sensitivity at the level of few per cent. For instance, the predicted day-night asymmetry of the SuperKamiokande signal is about $2 \%$ which is comparable with the existing $1 \sigma$ experimental error [6]. At SNO one expects the $2-4 \%$ asymmetry, consistent with the experimental result [3] at the $1 \sigma$ level.

Future experiments will have substantially higher sensitivity $[16,17,18,19]$. The solar neutrino studies enter a phase of precision measurements. 
In this connection it is important

- to give precise description of the LMA conversion, both in the Sun and in the Earth, taking into account various corrections;

- to estimate accuracy of the approximations made;

- to find the precise analytic expressions for probabilities and observables as functions of the oscillation parameters $\left(\Delta m^{2}, \sin ^{2} \theta_{12}\right)$. This will help to test the LMA solution and to search for physics beyond LMA.

We address these issues in the present paper. In section 2 we consider the non-adiabatic corrections to the LMA conversion probability. We calculate these corrections for propagation inside the Sun and the Earth. In section 3 we obtain the analytical formula for the probability averaged over the distribution of neutrino sources. In section 4 we derive the analytic formula for the $\nu_{e}$ regeneration effect in the Earth. We present our conclusions in Section 5 . In the appendices $\mathrm{A}$ and $\mathrm{B}$, alternative derivations of formulas for the regeneration factor are given.

\section{Non-adiabatic corrections to the LMA solution}

According to the LMA MSW solution of the solar neutrino problem, a conversion of the solar electron neutrinos is driven by mixing of the two active neutrinos, $\Psi_{f} \equiv\left(\nu_{e}, \nu_{a}\right)^{T}$ :

$$
\Psi_{f}=U(\theta) \Psi_{\text {mass }}
$$

where, in general, the mixing matrix is determined as

$$
U(\alpha)=\left(\begin{array}{cc}
\cos \alpha & \sin \alpha \\
-\sin \alpha & \cos \alpha
\end{array}\right)
$$

and $\Psi_{\text {mass }} \equiv\left(\nu_{1}, \nu_{2}\right)^{T}$ is the vector of mass states.

\subsection{LMA and Adiabaticity}

The main feature of the LMA solution is the adiabaticity of conversion. According to LMA the averaged $2 \nu$ survival probability of the electron neutrinos is given by the adiabatic formula $[2$, $20,21]$ :

$$
P_{e e}=\frac{1}{2}\left(1+\cos 2 \theta_{m}^{0} \cos 2 \theta\right)
$$

Here $\theta$ is the vacuum mixing angle, $\theta_{m}^{0}=\theta_{m}\left(x_{0}\right)$ is the mixing angle in matter in the neutrino production point, $x_{0}$, and the mixing angle in matter is determined by

$$
\cos 2 \theta_{m}(V)=\frac{\cos 2 \theta-2 E V / \Delta m^{2}}{\left[\left(\cos 2 \theta-2 E V / \Delta m^{2}\right)^{2}+\sin ^{2} 2 \theta\right]^{1 / 2}}, \quad V=\sqrt{2} G_{F} n_{e}(x) .
$$

Here $\Delta m^{2}$ is the mass squared difference, $E$ is the neutrino energy, $V$ is the potential, $G_{F}$ is the Fermi coupling constant and $n_{e}(x)$ is the number density of electrons in the point $x$. 
How precise the expression (3) is, and what are the non-adiabatic corrections? To answer these questions, we will elaborate on the adiabatic perturbation theory.

Dynamics of neutrino conversion is described in terms of the instantaneous eigenstates of the Hamiltonian in matter, $\nu_{1 m}, \nu_{2 m}$. Representing an arbitrary neutrino state as $|\nu\rangle=$ $\psi_{1 m}\left|\nu_{1 m}\right\rangle+\psi_{2 m}\left|\nu_{2 m}\right\rangle$, we can write the evolution equation in the base $\left(\nu_{1 m}, \nu_{2 m}\right)$ as $[2,20,22]$

$$
i \frac{d}{d x}\left(\begin{array}{c}
\psi_{1 m} \\
\psi_{2 m}
\end{array}\right)=\left(\begin{array}{cc}
-\frac{\Delta(x)}{4 E} & -i \dot{\theta}_{m}(x) \\
i \dot{\theta}_{m}(x) & \frac{\Delta(x)}{4 E}
\end{array}\right)\left(\begin{array}{l}
\psi_{1 m} \\
\psi_{2 m}
\end{array}\right),
$$

where

$$
\Delta(x) \equiv \Delta m^{2} \sqrt{\left(\cos 2 \theta-2 E V(x) / \Delta m^{2}\right)^{2}+\sin ^{2} 2 \theta}
$$

and

$$
\dot{\theta}_{m}(x) \equiv \frac{d \theta_{m}(x)}{d x}=\frac{E \Delta m^{2} \sin 2 \theta}{\Delta(x)^{2}} \frac{d V(x)}{d x} .
$$

The adiabatic approximation corresponds to a situation when

$$
\gamma \equiv \frac{4 E\left|\dot{\theta}_{m}\right|}{\Delta} \ll 1
$$

and the off-diagonal terms in the Hamiltonian (5) can be neglected. In this case there are no transitions between the eigenstates, and therefore the eigenstates propagate independently. The solution of (5) is straightforward:

$$
\Psi_{m}^{a d}(x)=S^{a d}\left(x, x_{0}\right) \Psi_{m}\left(x_{0}\right),
$$

where

$$
\Psi_{m}^{a d}(x)=\left(\begin{array}{c}
\psi_{1 m}^{a d}(x) \\
\psi_{2 m}^{a d}(x)
\end{array}\right), \quad \Psi_{m}\left(x_{0}\right)=\left(\begin{array}{c}
\psi_{1 m}\left(x_{0}\right) \\
\psi_{2 m}\left(x_{0}\right)
\end{array}\right)
$$

and the adiabatic evolution matrix is

$$
S^{a d}\left(x, x_{0}\right)=S^{a d}(\Phi)=\left(\begin{array}{cc}
e^{i \Phi(x)} & 0 \\
0 & e^{-i \Phi(x)}
\end{array}\right) .
$$

The adiabatic phase $\Phi(x)$ equals

$$
\Phi(x)=\frac{1}{4 E} \int_{x_{0}}^{x} d x^{\prime} \Delta\left(x^{\prime}\right) .
$$

A state initially produced as the electron neutrino, $\psi_{1 m}\left(x_{0}\right)=\cos \theta_{m}^{0}, \psi_{2 m}\left(x_{0}\right)=\sin \theta_{m}^{0}$, evolves as

$$
\nu(x)=\cos \theta_{m}^{0} e^{i \Phi(x)} \nu_{1 m}+\sin \theta_{m}^{0} e^{-i \Phi(x)} \nu_{2 m} .
$$

The incoherent survival probability (3) can be immediately obtained by averaging the matrix element squared, $\left|\left\langle\nu_{e} \mid \nu(x)\right\rangle\right|^{2}$, over the oscillations. 


\section{$2.2 \quad$ Non-adiabatic corrections}

The non-adiabatic corrections correspond to transitions between the instantaneous eigenstates. We calculate these corrections by solving the equation (5). We will implement a perturbation theory using the fact that for LMA the adiabaticity parameter (8) is very small.

Let us search for the solution of the equation (5) in the form

$$
\begin{aligned}
\left(\begin{array}{c}
\psi_{1 m}(x) \\
\psi_{2 m}(x)
\end{array}\right) & =\left(\begin{array}{cc}
1 & c(x) \\
-c^{*}(x) & 1
\end{array}\right)\left(\begin{array}{c}
\psi_{1 m}^{a d}(x) \\
\psi_{2 m}^{a d}(x)
\end{array}\right) \\
& =\left(\begin{array}{cc}
e^{i \Phi(x)} & c(x) e^{-i \Phi(x)} \\
-c^{*}(x) e^{i \Phi(x)} & e^{-i \Phi(x)}
\end{array}\right)\left(\begin{array}{l}
\psi_{1 m}\left(x_{0}\right) \\
\psi_{2 m}\left(x_{0}\right)
\end{array}\right),
\end{aligned}
$$

where $|c(x)| \ll 1$ is supposed to hold everywhere along the neutrino trajectory. (We check this a posteriori).

The expression (14) can be rewritten as

$$
\Psi_{m}(x)=S\left(x, x_{0}\right) \Psi_{m}\left(x_{0}\right)
$$

where the evolution matrix equals

$$
S\left(x, x_{0}\right) \equiv C S^{a d} \approx\left(\begin{array}{cc}
e^{i \Phi(x)} & c(x) e^{-i \Phi(x)} \\
-c^{*}(x) e^{i \Phi(x)} & e^{-i \Phi(x)}
\end{array}\right) .
$$

Inserting (14) into (5) we find the differential equation for $c(x)$ from the condition that the off-diagonal elements of the evolution equation for $\psi_{i m}$ are zero:

$$
i \frac{d}{d x} c(x)=-\frac{\Delta(x)}{2 E} c(x)-i \dot{\theta}_{m}(x) .
$$

Here the first order terms in $c(x)$ and $\dot{\theta}_{m}$ are kept only. In this approximation the energy gap between the states coincides with the adiabatic split $\Delta(x)$ given in Eq. (6).

The solution of equation (17) can be written in the following form

$$
c(x)=-\int_{x_{0}}^{x} d x^{\prime} \frac{d \theta_{m}\left(x^{\prime}\right)}{d x^{\prime}} \exp \left[-i \int_{x}^{x^{\prime}} d x^{\prime \prime} \frac{\Delta\left(x^{\prime \prime}\right)}{2 E}\right] .
$$

The integration constant is fixed by the condition : $c(x) \rightarrow 0$ as $\dot{\theta}_{m} \rightarrow 0$, so that $c\left(x_{0}\right)=0$.

Since for the LMA solution the phase $\Phi(x)$ is a fast oscillating function, the integral in (18) can be calculated using the following formula (essentially the integration by parts)

$$
\int_{a}^{b} f(x) e^{i g(x)} d x=\left.\left[-i \frac{f(x)}{g^{\prime}(x)}+\frac{f^{\prime}(x)}{g^{\prime 2}(x)}-\frac{f(x) g^{\prime \prime}(x)}{g^{\prime 3}(x)}\right] e^{i g(x)}\right|_{a} ^{b}+\mathcal{O}\left(1 / g^{\prime 3}\right)
$$

which is valid for smooth functions of $f(x)$ and $g(x)$. Here $g^{\prime}(x) \equiv d g(x) / d x$ and $f^{\prime}(x) \equiv$ $d f(x) / d x$. The formula gives very good approximation if $f(x) / g^{\prime}(x) \ll 1$. In the case of integral (18) this condition coincides with the adiabaticity condition (8) which is well satisfied. 
According to (19) and (18) we find

$$
\begin{aligned}
c\left(x_{f}\right) & =-\left.i \frac{2 E}{\Delta(x)} \frac{d \theta_{m}(x)}{d x} \exp \left[-i \int_{x_{f}}^{x} d x^{\prime} \frac{\Delta\left(x^{\prime}\right)}{2 E}\right]\right|_{x=x_{0}} ^{x=x_{f}} \\
& =-\left.i \operatorname{sign}\left(\dot{\theta}_{m}\right) \frac{\gamma(x)}{2} \exp \left[-2 i\left(\Phi(x)-\Phi\left(x_{f}\right)\right)\right]\right|_{x=x_{0}} ^{x=x_{f}},
\end{aligned}
$$

or explicitly

$$
c\left(x_{f}\right)=-\left.i \frac{2 E^{2} \Delta m^{2} \sin 2 \theta}{\Delta(x)^{3}} \frac{d V(x)}{d x} \exp \left[-i \int_{x_{f}}^{x} d x^{\prime} \frac{\Delta\left(x^{\prime}\right)}{2 E}\right]\right|_{x=x_{0}} ^{x=x_{f}},
$$

where $\operatorname{sign}\left(\dot{\theta}_{m}\right) \equiv \dot{\theta}_{m} /\left|\dot{\theta}_{m}\right|$.

We will apply this formula for neutrino propagation inside the Sun in section 2.3 and inside the Earth in section 4.

\subsection{Non-adiabatic corrections for propagation inside the Sun}

The survival probability with the adiabaticity violation effect included can be written as

$$
P_{e e}=\frac{1}{2}\left[1+\left(1-2 P_{c}\right) \cos 2 \theta_{m}^{0} \cos 2 \theta\right]
$$

where $P_{c}=\left|c\left(x_{f}\right)\right|^{2}$ is the jump probability - the probability of transition $\nu_{2 m} \rightarrow \nu_{1 m}$ on the way from $x_{0}$ to $x_{f}$.

Let us calculate $P_{c}$. Notice that for the LMA solution, one can not use the Landau-Zener formula $[23]$ for $P_{c}$ for the following reasons. The mixing angle in the final point of evolution, $x_{f}$, is large. The adiabaticity parameter $\left|4 E \dot{\theta}_{m} / \Delta\right|$ is of the same order for all points inside 0.3 of the solar radius [24]. The point of maximal adiabaticity violation is not the resonant point, though not far from it. Moreover, the resonance layer defined as $\left|2 E V-\Delta m^{2} \cos 2 \theta\right| \lesssim \Delta m^{2} \sin 2 \theta$, is broad since the vacuum mixing angle is large. Futhermore, significant part of the neutrino flux is produced inside the resonance region or does not cross the resonance region at all.

The double exponential formula [25] is not valid too. It requires production of neutrinos far above the resonance region in the density scale. This formula is not applied in the range

$$
\frac{\Delta m^{2}}{2 E} \cos 2 \theta \sim(1.6-8.0) \cdot 10^{-6} \frac{\mathrm{eV}^{2}}{\mathrm{MeV}}
$$

for which the density in the production point turns out to be close to the resonance density. For the best fit values of the LMA oscillation parameters the range $(23)$ corresponds to $E=(2-15)$ $\mathrm{MeV}$, that is, to the region of interest.

Let us apply the results of section 2.2 for calculation of the non-adiabatic corrections. Notice that at the surface of the Sun the effective potential $V$ is negligible and $\dot{\theta}_{m}$ can be taken zero. Using Eq. (21) we find the transition amplitude $c\left(x_{f}\right)$ on the way from the production point to the surface of the Sun in the leading order approximation as

$$
c\left(x_{f}\right)=\left.i \frac{2 E^{2} \Delta m^{2} \sin 2 \theta}{\Delta(x)^{3}} \frac{d V(x)}{d x}\right|_{x=x_{0}} \times \exp \left[i \int_{x_{0}}^{x_{f}} d x \frac{\Delta(x)}{2 E}\right] .
$$




\begin{tabular}{|c|c|c|c|c|c|c|c|c|}
\hline$K$ & $\mathrm{pp}$ & ${ }^{8} B$ & ${ }^{13} N$ & ${ }^{15} \mathrm{O}$ & ${ }^{17} F$ & ${ }^{7}$ Be & pep & hep \\
\hline$V_{K}\left(10^{-12} \mathrm{eV}\right)$ & 4.68 & 6.81 & 6.22 & 6.69 & 6.74 & 6.16 & 5.13 & 3.96 \\
\hline$\Delta V_{K}^{2} / V_{K}^{2}$ & 0.109 & 0.010 & 0.054 & 0.013 & 0.012 & 0.029 & 0.076 & 0.165 \\
\hline
\end{tabular}

Table 1: The average value of potential $\bar{V}_{K}$ and the corresponding value of $\Delta V_{K}^{2} / \bar{V}_{K}^{2}$ for different component of the solar neutrino spectrum.

Then the probability of non-adiabatic transition is given by

$$
P_{c}=\left|c\left(x_{f}\right)\right|^{2}=\frac{\gamma^{2}\left(x_{0}\right)}{4}=\frac{1}{16 \pi^{2}} \frac{l_{\text {osc }}^{2}\left(x_{0}\right)}{h^{2}\left(x_{0}\right)}\left[\frac{2 E V\left(x_{0}\right) \Delta m^{2} \sin 2 \theta}{\Delta\left(x_{0}\right)^{2}}\right]^{2},
$$

where

$$
h(x) \equiv V(x)\left[\frac{d V(x)}{d x}\right]^{-1}, \quad l_{o s c}(x) \equiv \frac{4 \pi E}{\Delta(x)}
$$

are the density height and the oscillation length in matter.

The transition probability $P_{c}(25)$ depends only on parameters of the initial (production) point. One can understand this by noting that $l_{o s c}(x) \ll h_{c}(x)$. Therefore many oscillation lengths are obtained on the distance in which the potential changes sizably. The non-adiabatic corrections are averaged out being negligible along the trajectory of the neutrino except for the boundaries of trajectory, i.e., around the production point or the point at the surface of the Sun. At the surface of the Sun the contribution can be neglected because the potential is zero.

The probability is determined basically by square of the ratio of the oscillation length and the density height. Second factor in (25) is of the order one. So, essentially $P_{c} \lesssim\left[l_{o s c}\left(x_{0}\right) / 4 \pi h\left(x_{0}\right)\right]^{2}$. Using the best fit values of the LMA oscillation parameters we find from (25)

$$
P_{c}=\left(10^{-9}-10^{-7}\right)\left(\frac{E}{10 \mathrm{MeV}}\right)^{2} \text {. }
$$

Here the numerical prefactor depends on the production point. As a function of $x_{0}$, the probability $P_{c}$ reaches maximum at around $(0.1-0.2) R_{\odot}$, where the potential doesn't drop down substantially and $h\left(x_{0}\right)$ reaches its almost minimal value due to increase of the density gradient. The corrections are negligible in the whole relevant range of neutrino energies and production points. The probability (27) strongly differs from what one would get using double-exponential formula [25]: $\sim e^{-4 \pi h / l_{o s c}} \lesssim 10^{-400}$.

Notice that the jump probability (25) equals (up to factor 4) the adiabaticity parameter in the production point squared, as is expected in the adiabatic perturbation theory. This contrasts the Landau-Zener probability, $P_{c} \sim \exp (-\pi / 2 \gamma)$, which is essentially non-perturbative effect.

\section{Averaging over production region: analytic results}

In the adiabatic approximation the survival probability depends on the potential (density) in neutrino production point $r$ :

$$
P_{e e}=P_{e e}\left(V_{0}\right), \quad V_{0}=V\left(x_{0}=r\right) .
$$


Observables at the Earth are determined by the survival probability averaged over the neutrino production region:

$$
P_{K}=\frac{\int d r G_{K}(r) P_{e e}(r)}{\int d r G_{K}(r)}, \quad K=p p, p e p, B e, N, O, F, B, h e p,
$$

where $G_{K}(r)$ is the distribution of sources of the $K$ component of neutrino spectrum. The distributions are different for different components.

Let us introduce the average value of the potential in the production region for the type $K$ neutrinos:

$$
\bar{V}_{K} \equiv \frac{\int d r G_{K}(r) V(r)}{\int d r G_{K}(r)}
$$

We will use the fact that in the effective production region, $V(r)$ deviates weakly from $\bar{V}_{K}$. Therefore the survival probability can be expanded in series around $\bar{V}_{K}$ :

$$
P_{e e}=P_{e e}\left(\bar{V}_{K}\right)+\left(\frac{d P_{e e}}{d V}\right)_{V=\bar{V}_{K}}\left(V-\bar{V}_{K}\right)+\frac{1}{2}\left(\frac{d^{2} P_{e e}}{d V^{2}}\right)_{V=\bar{V}_{K}}\left(V-\bar{V}_{K}\right)^{2}+\cdots
$$

Inserting this expression into (29) and using the definition (30) we find

$$
P_{K}=P_{e e}\left(\bar{V}_{K}\right)-\frac{3 E^{2}}{\left(\Delta m^{2}\right)^{2}} \frac{\sin ^{2} 2 \theta \cos 2 \theta\left(\cos 2 \theta-2 E \bar{V}_{K} / \Delta m^{2}\right)}{\left[\left(\cos 2 \theta-2 E \bar{V}_{K} / \Delta m^{2}\right)^{2}+\sin ^{2} 2 \theta\right]^{5 / 2}} \Delta V_{K}^{2},
$$

where

$$
\Delta V_{K}^{2} \equiv \frac{\int d r G_{K}(r)\left(V(r)-\bar{V}_{K}\right)^{2}}{\int d r G_{K}(r)}
$$

Notice that the correction to $P_{e e}$ appears in the second order of the deviation of potential from the average value. The expression for probability (32) can be rewritten as

$$
P_{K}=\frac{1}{2}+\frac{1}{2}\left(1-\delta_{K}\right) \cos 2 \theta_{m}\left(\bar{V}_{K}\right) \cos 2 \theta,
$$

where the correction $\delta_{K}$ equals

$$
\delta_{K}=\frac{3}{2} \frac{\left(2 E \bar{V}_{K} / \Delta m^{2}\right)^{2} \sin ^{2} 2 \theta}{\left[\left(\cos 2 \theta-2 E \bar{V}_{K} / \Delta m^{2}\right)^{2}+\sin ^{2} 2 \theta\right]^{2}} \frac{\Delta V_{K}^{2}}{\bar{V}_{K}^{2}} .
$$

In the Table 1 we present the average values of potentials and the corresponding second order deviations from the average values for eight types of solar neutrinos. We use the distributions of neutrino sources from the BP2000 model [26]. The expansion parameters $\Delta V_{K}^{2} / \bar{V}_{K}^{2}$ are all small, especially for the boron neutrinos which have the narrowest distribution of sources.

In Fig. 1 we compare the probability $P_{K}$ obtained from the approximate analytic formula (34) with results of numerical calculations, $P_{K}^{\prime}$. $P_{K} / P_{K}^{\prime}-1$ is shown as a function of $E / \Delta m^{2}$. The lines have been cut for $K=p p, B e 7$, pep, $N, O, F$ because of their lower energies in comparison with the energies of hep and boron neutrinos. The plot shows that the analytic formula is rather precise. In particular, the deviations are extremely small $\left(\lesssim 10^{-3}\right)$ for small and large values of $E / \Delta m^{2}$. Relatively large deviations can be seen in the intermediate region of $E / \Delta m^{2}$. For example, for $K=$ hep the magnitude of $P_{K} / P_{K}^{\prime}-1$ reaches maximum $1.8 \%$ at around $E / \Delta m^{2} \approx 34 \times 10^{10} \mathrm{eV}^{-1}$. The corrections $\delta_{K}$ are important: e.g., for the hep neutrinos the deviation would be up to $6 \%$ without $\delta_{K}$. 


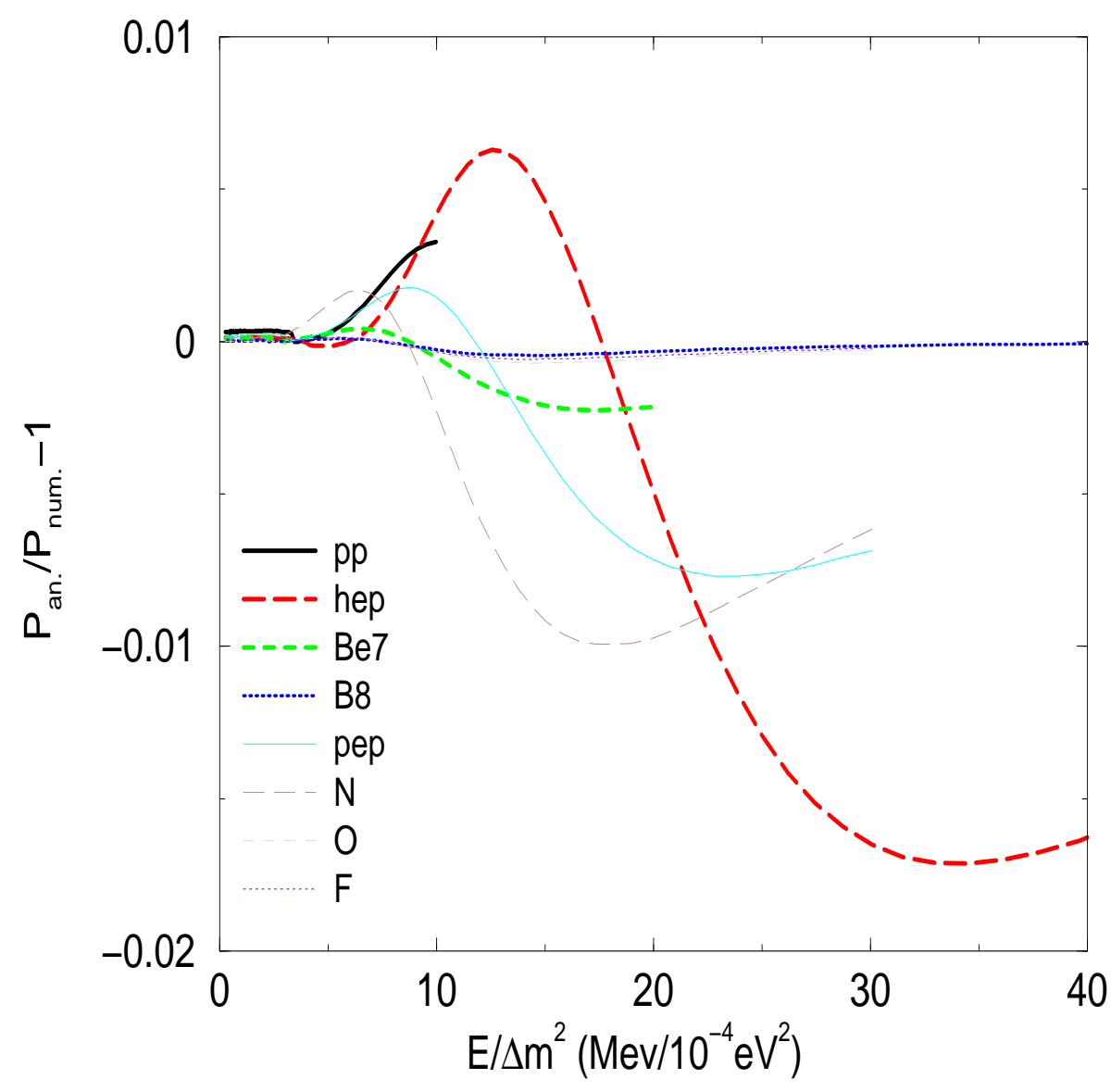

Figure 1: Deviations of the probability $P_{K}$ given in formula (34) from the numerically calculated probability, $P_{K}^{\prime}$ for different components of the solar neutrino spectrum.

\section{The Earth matter effect: analytic study}

The solar neutrinos arrive at the surface of the Earth as incoherent fluxes of the mass states. The mass states oscillate in the matter of the Earth producing partial regeneration of the electron neutrino flux [27, 28, 29, 30, 31, 32, 33, 34]. Previously the effect has been described in one or two layers approximation. In the later case, interference effects of contributions from the core and the mantle have been discussed [33,34]. In this section we will study effects for the realistic density profile of the Earth.

\subsection{Regeneration factor and the Earth density profile}

The probability of $\nu_{2} \rightarrow \nu_{e}$ transition can be written as

$$
P\left(\nu_{2} \rightarrow \nu_{e}\right) \equiv \sin ^{2} \theta+f_{\text {reg }},
$$

where $f_{\text {reg }}$ is the regeneration factor which describes the Earth matter effect. In the absence of matter (i.e., during the day) $f_{r e g}=0$. Using the definition (36) we find the $\nu_{e}$ survival probability with the regeneration effect included as

$$
P_{e e}=\frac{1}{2}\left(1+\cos 2 \theta_{m}^{0} \cos 2 \theta\right)-\cos 2 \theta_{m}^{0} f_{r e g} .
$$




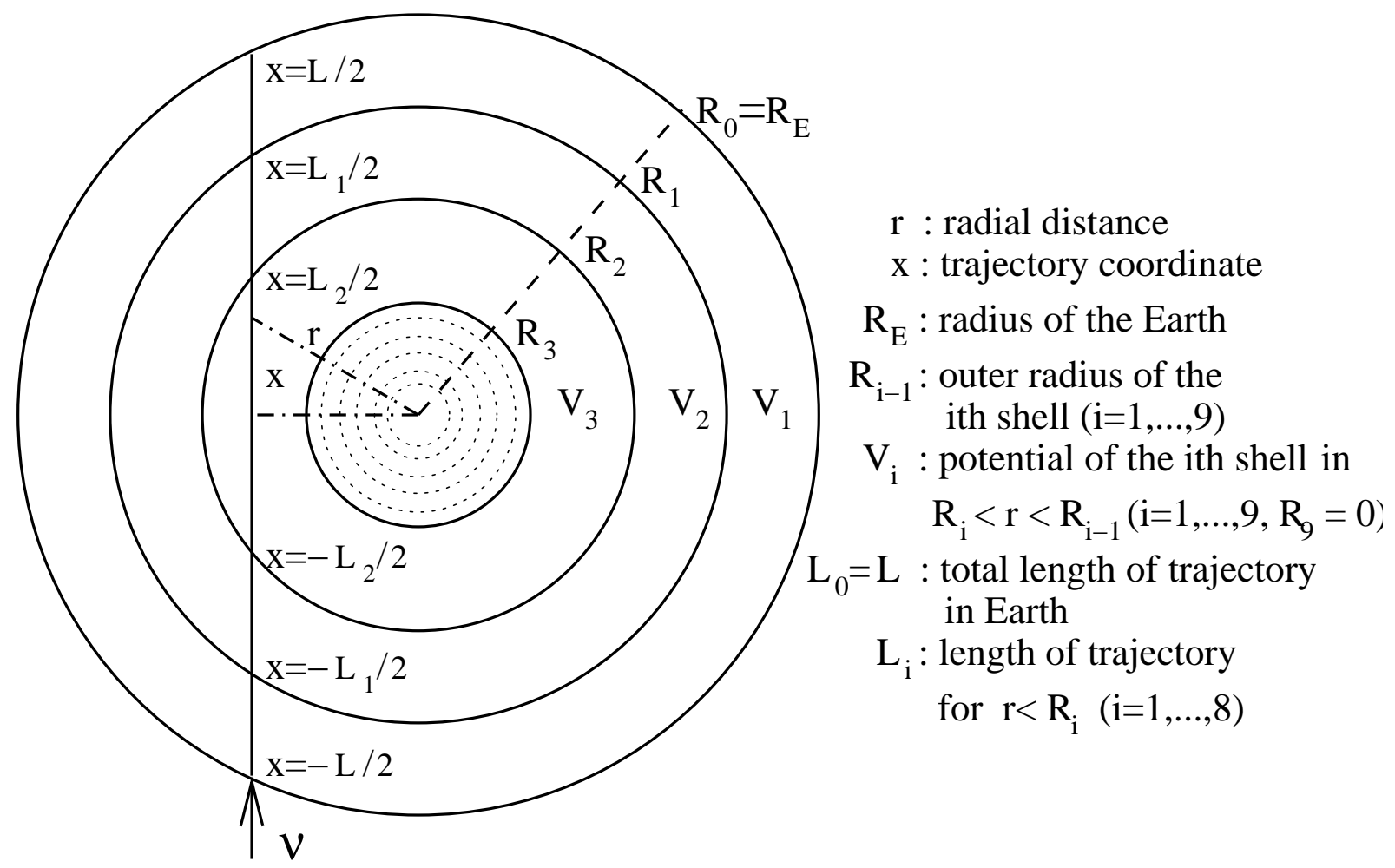

Figure 2: Structure of the Earth density profile. We indicate notations used in the text.

Notice that the mixing angle $\theta_{m}^{0}$ in the neutrino production point in the Sun determines the mass $\left(\nu_{1}, \nu_{2}\right)$ composition of the neutrino flux which arrives at the Earth.

The essential feature of the LMA solution is that the Earth matter effect is small. This smallness is characterized by the ratio

$$
\eta \equiv \frac{2 E V}{\Delta m^{2}}=0.024\left(\frac{E}{10 \mathrm{MeV}}\right)\left(\frac{6.3 \times 10^{-5} \mathrm{eV}^{2}}{\Delta m^{2}}\right) \frac{V}{V_{A}}, \quad V_{A}=\sqrt{2} G_{F} N_{A},
$$

where $N_{A}$ is the Avogadro number. We will use $\eta$ as the expansion parameter.

The $\nu_{2} \rightarrow \nu_{e}$ transition probability can be written as

$$
\begin{aligned}
P\left(\nu_{2} \rightarrow \nu_{e}\right) & =\left|\left\langle\nu_{e}\left|U\left(\theta_{m R}\right) S\left(x_{f}, x_{0}\right) U^{\dagger}\left(\theta_{m R}\right) U(\theta)\right| \nu_{2}\right\rangle\right|^{2} \\
& =\left|\left\langle\nu_{e}\left|U\left(\theta_{m R}\right) S\left(x_{f}, x_{0}\right) U^{\dagger}\left(\theta_{m R}-\theta\right)\right| \nu_{2}\right\rangle\right|^{2},
\end{aligned}
$$

where $\theta_{m R}$ is the mixing angle in matter at the surface of the Earth and the matrix $S\left(x_{f}, x_{0}\right)$ given in (16) describes evolution of the neutrino eigenstates in matter. Noting that in matter of the Earth

$$
\sin \left(\theta_{m R}-\theta\right) \approx \frac{E V_{R}}{\Delta m^{2}} \sin 2 \theta \ll 1,
$$

we find from (14), (36) and (39) an expression for the regeneration factor in the lowest order in $c(x)$ and $\sin \left(\theta_{m R}-\theta\right)$ as

$$
f_{\text {reg }}=\frac{2 E V_{R}}{\Delta m^{2}} \sin ^{2} 2 \theta \sin ^{2} \Phi\left(x_{f}\right)+\sin 2 \theta \operatorname{Re}\left\{c\left(x_{f}\right)\right\}
$$


where $V_{R}$ is the potential at the surface of the Earth and $\Phi\left(x_{f}\right)$ is the total phase acquired along the trajectory in the Earth.

For the profile with slowly changing density (lowest adiabatic approximation), $c \approx 0$, we obtain

$$
f_{\text {reg }}^{a d}=\frac{2 E V_{R}}{\Delta m^{2}} \sin ^{2} 2 \theta \sin ^{2} \Phi\left(x_{f}\right) .
$$

The prefactor (the depth of oscillations) is determined by the potential at the surface of the Earth, whereas the phase is given by the integral along whole trajectory. For one layer with constant potential (density), and therefore $c=0$, the regeneration factor (41) or (42) is reduced to the well known expression:

$$
f_{\text {reg }}=\frac{2 E V_{R}}{\Delta m^{2}} \sin ^{2} 2 \theta \sin ^{2} \frac{\pi L}{l_{m}} .
$$

Here $L$ is the distance traveled by neutrino in the Earth and $l_{m}$ is the oscillation length in matter.

Let us consider a neutrino propagation in realistic density profile of the Earth. The profile can be described by $n$ nearly spherical shells of matter with sharp (step-like) density changes at the borders of shells and slow variation of density in layers between the borders. According to the PREM model $n=9$ [35]. So, in $i$ th shell $(i=1, \cdots, n)$, the potential $V_{i}$ is a smooth function of the radial distance $r$. Crossing $j$ shells corresponds to crossing $2 j-1$ layers (see Fig. 2). We denote by $R_{i-1}$ the outer radius of $i$ th shell, so that $R_{0}$ corresponds to the radius of the Earth: $R_{0}=R_{E}$.

The trajectory of the neutrino is characterized by the zenith angle $\theta_{Z}$. We determine a position of neutrino along trajectory by the coordinate $x$ with origin in the center of trajectory, so that

$$
x \in[-L / 2, L / 2], \quad x^{2}=r^{2}-R_{E}^{2} \sin ^{2} \theta_{Z} .
$$

Here $L$ is the total length of the trajectory in the Earth. The length of the part of trajectory inside border $R_{i}$ is given by

$$
L_{i}=\sqrt{R_{i}^{2}-R_{E}^{2} \sin ^{2} \theta_{Z}}
$$

and $L_{0}=L$ by definition.

We introduce the adiabatic phase $\Phi_{i}$ acquired by neutrinos in the interval $-L_{i} / 2 \leq x \leq L_{i} / 2$ along the trajectory, that is, inside the outer border of the $(i+1)$ th shell:

$$
\begin{aligned}
\Phi_{i} & =\int_{-L_{i} / 2}^{L_{i} / 2} d x \frac{\Delta(x)}{4 E} \\
& \approx \int_{-L_{i} / 2}^{L_{i} / 2} d x\left[\frac{\Delta m^{2}}{4 E}-\frac{1}{2} \cos 2 \theta V(x)+\frac{E \sin ^{2} 2 \theta}{2 \Delta m^{2}} V^{2}(x)\right], \quad i=0, \cdots, n-1 .
\end{aligned}
$$

$\Delta(x)$ is given in Eq. (6). Here we keep the order $V^{2}$ term since due to integration its contribution to the phase is not negligible. 
At the borders of shells there are jumps of the potential and hence the discontinuities of the mixing angle in matter. We denote them as

$$
\begin{aligned}
\Delta V_{i} & \equiv V_{i+1}\left(R_{i}\right)-V_{i}\left(R_{i}\right), \quad i=0, \cdots, n-1, \\
\Delta \theta_{m i} & \equiv \theta_{m}\left(V_{i+1}\left(R_{i}\right)\right)-\theta_{m}\left(V_{i}\left(R_{i}\right)\right), \quad i=0, \cdots, n-1 .
\end{aligned}
$$

At the surface of the Earth, we obtain

$$
\Delta V_{0}=V_{R}, \quad \Delta \theta_{m 0}=\theta_{m R}-\theta .
$$

Furthermore, we find

$$
\sin \Delta \theta_{m i} \approx \frac{E \Delta V_{i}}{\Delta m^{2}} \sin 2 \theta, \quad \cos \Delta \theta_{m i} \approx 1, \quad i=0, \cdots, n-1 .
$$

Corrections to (50) are of the order $\eta^{2}$, and hence negligible.

Smooth variation of the potential in each shell of the Earth can be approximated by the analytic formula [36]:

$$
V_{i}=V_{A}\left[\alpha_{i}+\beta_{i} \frac{r^{2}}{R_{E}^{2}}+\gamma_{i} \frac{r^{4}}{R_{E}^{4}}\right], \quad i=1, \cdots, n .
$$

Let us find an analytic expression for the regeneration factor using the density profile described above. The problem can be solved in two steps: (1) computation of the non-adiabatic corrections to propagation within a given layer, $\Delta f_{i}$; $(2)$ computation of effect of the borders between layers, $\Delta f_{i}^{\text {jump }}$. So that

$$
f_{r e g}=f_{r e g}^{a d}+\sum_{i} \Delta f_{i}+\sum_{i} \Delta f_{i}^{j u m p} .
$$

The virtue of the LMA solution is that it enables us to study both effects using the same formalism of the adiabatic perturbation theory.

\subsection{Non-adiabatic corrections in a layer of the Earth}

Let us compute the non-adiabatic corrections for one layer. Suppose a neutrino trajectory crosses the $i$ th layer with the borders at $x=L_{i} / 2$ and $x=L_{i-1} / 2$. Using (44) and (51), the potential in this layer can be expressed in terms of the trajectory coordinate as

$$
V_{i}=V_{A}\left[\alpha_{i}^{\prime}+\beta_{i}^{\prime} \frac{x^{2}}{R_{E}^{2}}+\gamma_{i}^{\prime} \frac{x^{4}}{R_{E}^{4}}\right] .
$$

Here

$$
\alpha_{i}^{\prime}=\alpha_{i}+\beta_{i} \sin ^{2} \theta_{Z}+\gamma_{i} \sin ^{4} \theta_{Z}, \quad \beta_{i}^{\prime}=\beta_{i}+2 \gamma_{i} \sin ^{2} \theta_{Z}, \quad \gamma_{i}^{\prime}=\gamma_{i} .
$$

According to (45) and (54) the gradients of potential at the borders equal

$$
\begin{gathered}
\left.\frac{d V_{i}(x)}{d x}\right|_{x=\frac{L_{i}}{2}}=\frac{2 V_{A}}{R_{E}} \sqrt{y_{i}^{2}-\sin ^{2} \theta_{Z}}\left(\beta_{i}+2 \gamma_{i} y_{i}^{2}\right), \\
\left.\frac{d V_{i}(x)}{d x}\right|_{x=\frac{L_{i-1}}{2}}=\frac{2 V_{A}}{R_{E}} \sqrt{y_{i-1}^{2}-\sin ^{2} \theta_{Z}}\left(\beta_{i}+2 \gamma_{i} y_{i-1}^{2}\right) .
\end{gathered}
$$


where $y_{i} \equiv R_{i} / R_{E}$. Then for this layer Eq. (21) gives the amplitude of non-adiabatic transition

$$
\begin{aligned}
& c_{i}=-\left.2 i e^{2 i \Phi_{0}} \frac{E^{2} \Delta m^{2} \sin 2 \theta}{\Delta^{3}(x)} \frac{d V(x)}{d x} e^{-2 i \Phi(x)}\right|_{x=\frac{L_{i}}{2}} ^{x=\frac{L_{i-1}}{2}} \\
& =-\frac{4 i E^{2} \sin 2 \theta}{\left(\Delta m^{2}\right)^{2} R_{E}} V_{A} e^{i \Phi_{0}}\left[\left(\beta_{i}+2 \gamma_{i} y_{i-1}^{2}\right) \sqrt{y_{i-1}^{2}-\sin ^{2} \theta_{Z}} e^{-i \Phi_{i-1}}\right. \\
& \left.-\left(\beta_{i}+2 \gamma_{i} y_{i}^{2}\right) \sqrt{y_{i}^{2}-\sin ^{2} \theta_{Z}} e^{-i \Phi_{i}}\right]
\end{aligned}
$$

where phases $\Phi_{i}$ are defined in (46). Inserting this expression into (41) we obtain the nonadiabatic correction from this layer, $\Delta f_{i}$ to the regeneration factor as

$$
\begin{aligned}
\Delta f_{i} & =\frac{4 E^{2} \sin ^{2} 2 \theta}{\left(\Delta m^{2}\right)^{2} R_{E}} V_{A}\left[\left(\beta_{i}+2 \gamma_{i} y_{i-1}^{2}\right) \sqrt{y_{i-1}^{2}-\sin ^{2} \theta_{Z}} \sin \left(\Phi_{0}-\Phi_{i-1}\right)\right. \\
& \left.-\left(\beta_{i}+2 \gamma_{i} y_{i}^{2}\right) \sqrt{y_{i}^{2}-\sin ^{2} \theta_{Z}} \sin \left(\Phi_{0}-\Phi_{i}\right)\right] .
\end{aligned}
$$

The ratio of the absolute value of correction and the adiabatic term equals

$$
\frac{\left|\Delta f_{i}\right|}{\left|f_{\text {reg }}^{\text {ad }}\right|} \sim \frac{2 E}{\Delta m^{2} \sin 2 \theta R_{E}} \frac{V_{A}\left(\beta_{i}+2 \gamma_{i} y_{i}^{2}\right)}{V_{R}} \sqrt{y_{i}^{2}-\sin ^{2} \theta_{Z}} \sim \frac{2 E}{\Delta m^{2}} \frac{1}{R_{E}},
$$

where $R_{E}$ plays the role of typical scale of the density change. As an example, let us consider the layer between $0.895 R_{E}$ and $0.546 R_{E}$. In this layer $\alpha=3.156, \beta=-1.459$ and $\gamma=0.280$ [36]. From (59) we obtain that the non-adiabatic correction to the regeneration factor is about $(1-2) \%$ at $E=10 \mathrm{MeV}$.

Notice that for some particular values of energies and $\theta_{Z}$, the contributions $\Delta f_{i}$ from different layers $i$ may sum up "constructively" producing larger effect. In this connection let us notice the following.

1) The enhancement effect may occur for exceptional values of $E$ and $\theta_{Z}$ and therefore any realistic averaging over $E$ and integration over $\theta_{Z}$ will wash it out.

2) The enhancement can not be large (proportional to the number of layers, $n$ ) since (i) only few layers give significant contribution and for the rest, the effect is below 1\%; (ii) there is a systematic cancellation of contributions from the upper limit of integration in $\Delta f_{i}$ and the lower limit of integration in $\Delta f_{i-1}$ (the adiabatic phases are the same for both); (iii) typically, contributions from two layers of the same shell have opposite signs.

So, we conclude that the non-adiabaticity within layers of the Earth can be safely neglected.

\subsection{Effects of several layers}

The jumps of potential between the layers strongly violate the adiabaticity and on the first

glance, the adiabatic perturbation theory can not be applied. We show, however, that the results for non-adiabatic case obtained in section 2.2 can be also used here. The key point is that for the LMA parameters the Earth matter effects are small, whatever the density profile in the Earth is. As a consequence, variations of the mixing angle in matter are small: $\left|\Delta \theta_{m}\right| \ll \theta$, and essentially the expansion parameter here is $\eta$. 


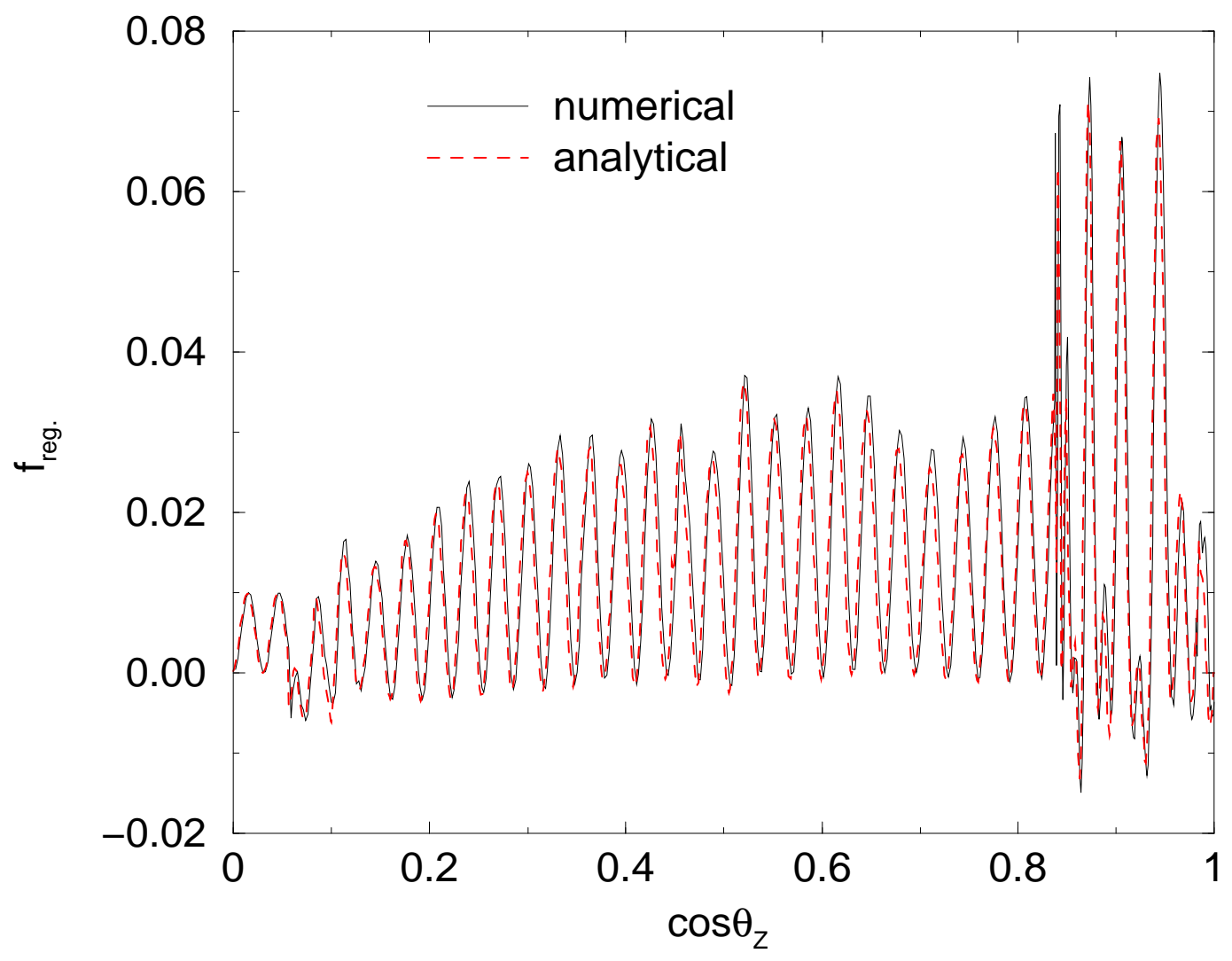

Figure 3: The regeneration factor as function of $\cos \theta_{Z}$ for $E=10 \mathrm{MeV}, \Delta m^{2}=6.3 \times 10^{-5} \mathrm{eV}^{2}$, and $\tan ^{2} \theta=0.4$. We compare result of numerical computations with analytic result (63) for the PREM model.

Consider a neutrino trajectory which crosses $2 n-1$ layers ( $n$ shells). In the points of the trajectory $x=\mp L_{i} / 2$ neutrinos cross the borders of shell with $r=R_{i}$, as illustrated in Fig. 2. The corresponding potential jumps equal $\pm \Delta V_{i}$ for $x=\mp L_{i} / 2$. Using the expression (7) we obtain in the lowest approximation

$$
\dot{\theta}_{m}(x)=\frac{E \sin 2 \theta}{\Delta m^{2}} \sum_{i=1}^{n-1} \Delta V_{i}\left[\delta\left(x+\frac{L_{i}}{2}\right)-\delta\left(x-\frac{L_{i}}{2}\right)\right] .
$$

As it has been shown in section 4.2 to a good approximation one can take $\dot{\theta}_{m}=0$ everywhere outside the borders.

The evolution equation (5) can be averaged in small intervals $\Delta x \ll 1 / \Delta(x)$ to eliminate $\delta$-functions which originate from $\dot{\theta}_{m}$. However, this is not necessary since in the expression for $c(x)$ in $(17) \dot{\theta}_{m}$ is integrated anyway.

Plugging expression (60) into Eq. (18) we obtain

$$
c(L / 2)=\frac{E \sin 2 \theta}{\Delta m^{2}} e^{i \Phi_{0}} \sum_{i=1}^{n-1} \Delta V_{i}\left(e^{-i \Phi_{i}}-e^{i \Phi_{i}}\right),
$$

where $\Phi_{i}$ are defined in (46). 
Inserting the real part of $c(L / 2)$ into (41) we find the regeneration factor for the case of $n$ shells crossing:

$$
f_{r e g}=\frac{2 E V_{R}}{\Delta m^{2}} \sin ^{2} 2 \theta \sin ^{2} \Phi_{0}+\sum_{i=1}^{n-1} \frac{2 E \Delta V_{i}}{\Delta m^{2}} \sin ^{2} 2 \theta \sin \Phi_{i} \sin \Phi_{0} .
$$

In the Appendix A we present the rigorous derivation of this factor considering evolution in the sequent layers explicitly. The results of two approaches coincide exactly in the first order in $E V / \Delta m^{2}$.

Noting that $V_{R}=\Delta V_{0}$ is the jump of potential at the surface of the Earth, we can rewrite the expression (62) in the following compact form:

$$
f_{\text {reg }}=\frac{2 E \sin ^{2} 2 \theta}{\Delta m^{2}} \sin \Phi_{0} \sum_{i=0}^{n-1} \Delta V_{i} \sin \Phi_{i} .
$$

So, $f_{\text {reg }}$ is proportional to the sum of similar terms which correspond to the borders of the shells. Each term is the product of the potential jump at a given border and sine of the total adiabatic phase acquired on the part of trajectory inside a given border (that is, from $-L_{i} / 2$ to $L_{i} / 2$ for the border $i$ ). The sum runs over all borders including the surface of the Earth. The expression (63) corresponds to the symmetric density profile. The zenith angle dependence of the regeneration factor appears via the phases: $\Phi_{i} \equiv \Phi_{i}\left(\theta_{Z}\right)$.

The formula (63) (which is the main result of our study) allows us to get complete understanding of the Earth matter effects including effects of complicated shell structure. Apparently, this is not possible using the one layer approximation (42), where the interference terms induced by different shells are absent.

In Fig. 3 we compare the zenith angle dependence of the regeneration factor computed using the analytic formula (62) with the one obtained by the exact numerical integration for the PREM profile. Two results coincide extremely well. One can see that the analytic formula reproduces quite precisely the magnitude and the phase structure of the regeneration factor.

Let us mark some features. The change of the oscillatory behaviors for $\cos \theta_{Z} \gtrsim 0.83$ is induced by the sharp density jump at the border between the mantle and the core of the Earth, at $r=0.54 R_{E}$. Notice that at $\cos \theta_{Z} \gtrsim 0.83$ the amplitude of oscillations for some periods increases, however, the frequency of large peaks becomes lower. So that the average value of $f_{\text {reg }}$ does not increase in comparison with the value for $\cos \theta_{Z}<0.83$.

For small $\cos \theta_{Z}$ the dependence of $f_{\text {reg }}$ on $\theta_{Z}$ is a result of interference of terms in (63) which correspond to the outer shells of the Earth. To understand this dependence, it is convenient to introduce the phase $\varphi_{i}$ :

$$
\varphi_{i} \equiv \Phi_{0}-\Phi_{i}
$$

so that $\varphi_{i} / 2$ is the phase acquired by neutrino on the way from the surface of the Earth to $r=R_{i}$. Using $\varphi_{i}$ we can rewrite the expression for regeneration factor (63) as

$$
f_{r e g}=\frac{2 E \sin ^{2} 2 \theta}{\Delta m^{2}} \sum_{i=0}^{n-1} \Delta V_{i}\left[\sin ^{2} \Phi_{0} \cos \varphi_{i}-\frac{1}{2} \sin 2 \Phi_{0} \sin \varphi_{i}\right] .
$$

Apparently, if averaging over $\varphi_{i}$ occurs only the term with $i=0$ survives $\left(\varphi_{0}=0\right)$ in $f_{r e g}$ which is reduced to the adiabatic expression for one layer. 


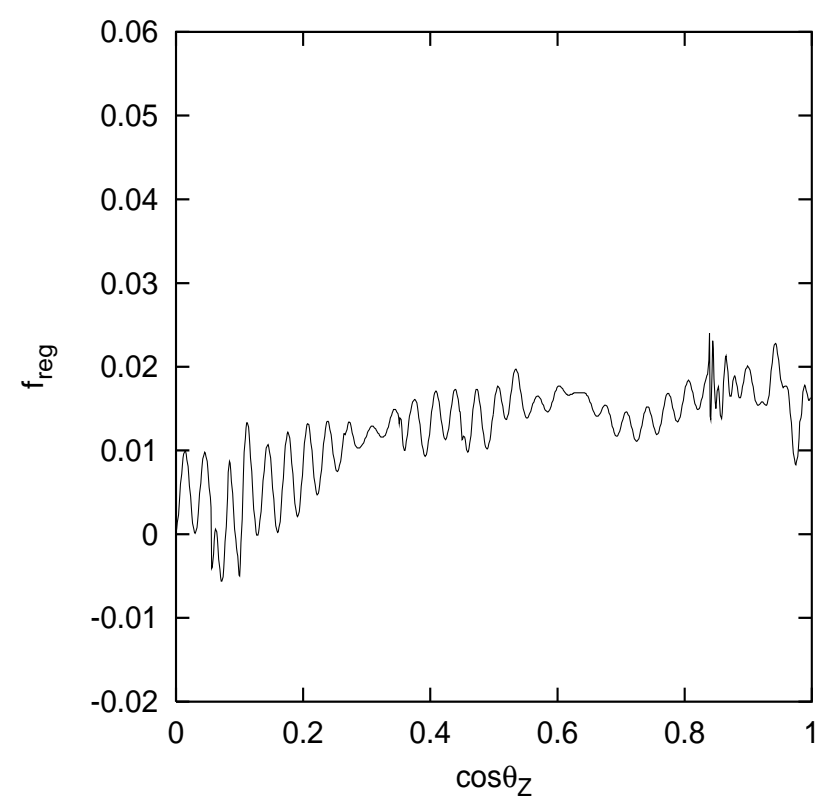

(a)

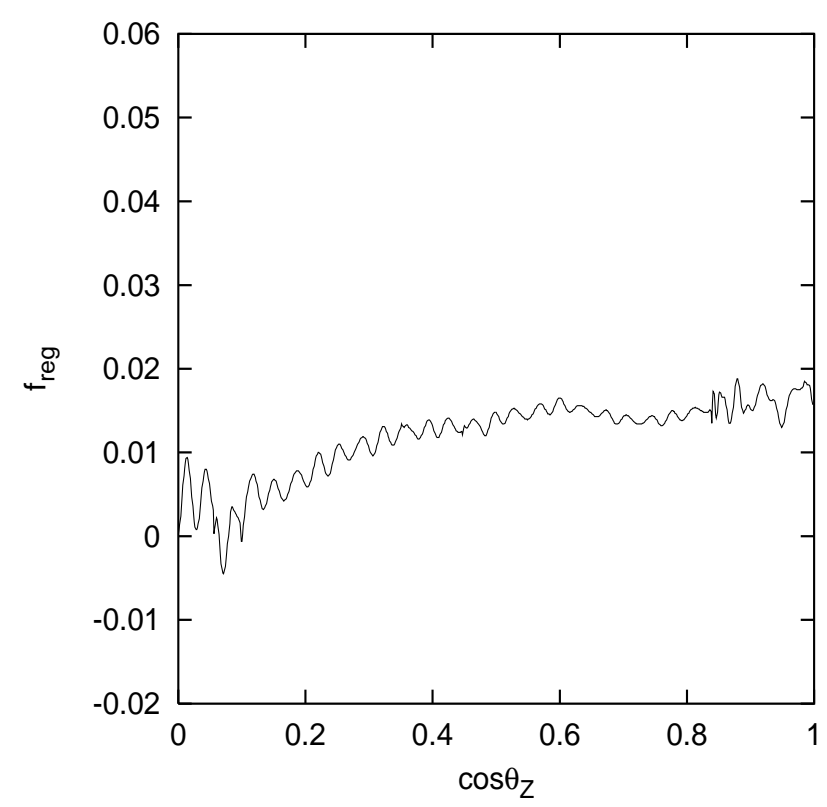

(b)

Figure 4: The regeneration factor averaged over the energy intervals (a) $E=(9.5-10.5) \mathrm{MeV}$; (b) $E=(8-11) \mathrm{MeV}$. For oscillation parameters we take $\Delta m^{2}=6.3 \times 10^{-5} \mathrm{eV}^{2}$ and $\tan ^{2} \theta=0.4$.

The increase of regeneration factor with $\cos \theta_{Z}$ in the range $0.2-0.5$ is related to the effect of three density jumps near the surface of the Earth: according to the PREM profile they are situated at depths $10 \mathrm{~km}, 22 \mathrm{~km}$ and $31 \mathrm{~km}$ correspondingly. The distance, $L-L_{i}$, on which $\varphi_{i}$ is acquired depends on the zenith angle as

$$
L-L_{i} \approx 2 \frac{R_{E}-R_{i}}{\cos \theta_{Z}}, \quad \cos \theta_{Z}>\sqrt{1-R_{i}^{2} / R_{E}^{2}}
$$

( $R_{E}-R_{i}$ is the depth from the surface of the Earth to the borders of the $i$ th shell). In the case of small $\cos \theta_{Z}\left(\cos \theta_{Z} \lesssim 0.2\right)$, the distance $L-L_{i}$ can be of several hundreds kilometers which is comparable to or larger than the oscillation length. Furthermore, $L-L_{i}$ and $\varphi_{i}$ are fast changing functions of $\theta_{Z}$. So, $\varphi_{i}$ are large and different for different $i$. Therefore, the terms $\sin \Phi_{0} \sin \Phi_{i}$ for different $i(i=1,2,3)$ are quite different and therefore partially cancel each other ("interfere destructively").

In constrast, for $\cos \theta_{Z} \gtrsim 0.5$, the distances $L-L_{i}(i=1,2,3)$ for the outer shells become much smaller than the oscillation length and they slowly change with $\cos \theta_{Z}$. In this case the phases $\varphi_{i}(i=1,2,3)$ are all small and $\sin \Phi_{0} \sin \Phi_{i} \approx \sin ^{2} \Phi_{0}$. So, for $\cos \theta_{Z}>0.5$ the effects of outer shells "interfere constructively" producing larger regeneration factor. It is then possible to account these close layers effectively as a single layer, as it was done in Ref. [36]. Increase of the regeneration factor in the transition region $\cos \theta_{Z}=(0.2-0.5)$ corresponds to convergence of the term $\sin \Phi_{0} \sin \Phi_{i}(i=1,2,3)$ to $\sin ^{2} \Phi_{0}$.

\subsection{Averaging over the neutrino energy}

For the LMA solution the oscillation length in the Earth is small: $l_{m} \approx l_{\nu} \ll R_{E}$. Since the time of the neutrino detection is well known, averaging over the zenith angle can be avoided, 
and in fact, in the unbinned analysis of the data developed recently [6] one needs to know the zenith angle dependence without averaging. At the same time since the recoil electron (and not neutrino) energy is measured and a detector has finite energy resolution, averaging over the neutrino energy occurs.

In the leading approximation the phase equals $\varphi_{i} \approx \Delta m^{2}\left(L-L_{i}\right) /(4 E)$. Therefore the energy resolution $\Delta E$ corresponds to averaging over the interval of phases:

$$
\Delta \varphi_{i} \approx \varphi_{i} \frac{\Delta E}{E} .
$$

If $L-L_{i} \gg l_{m}$, so that $\varphi_{i} \gg 1$, the interval $\Delta \varphi_{i}$ can be large, thus leading to strong averaging of terms $\sin \Phi_{0} \sin \Phi_{i}$ in (63). This happens to the contributions from structures situated far from the surface of the Earth. In Fig. 4 we show the result of averaging of the regeneration factor folded with the cross section of the neutrino-electron elastic scattering over two different energy intervals. Comparing with Fig. 3, one sees that the complicated oscillatory pattern produced by the density jumps in the central regions of the Earth is strongly averaged when $\cos \theta_{Z}$ is large (for general analysis of this effect see [37]). According to Fig. 4 for $\cos \theta_{Z} \gtrsim 0.4$ the regeneration factor oscillates with small depth around $f_{\text {reg }} \approx 1.5 \%$. This happens because the main term $\sin ^{2} \Phi_{0}$ is strongly averaged too.

In contrast, for $\cos \theta_{Z} \lesssim 0.2$ only the outer structures of the Earth can contribute and the averaging is not as efficient as for large $\cos \theta_{Z}$. Indeed, for the borders of outer shells

$(i=1,2,3)$ the distance where $\varphi_{i}$ is acquired, $L-L_{i} \leq 2 R_{E} \sqrt{1-R_{i}^{2} / R_{E}^{2}}$, can be about several hundreds kilometers, that is, comparable with the oscillation length in matter. In this case the phase interval $\Delta \varphi_{i} \approx \varphi_{i} \Delta E / E$ is still not large enough to give sufficient averaging. For $\cos \theta_{Z}<0.4, \Delta \Phi_{0} \approx \Phi_{0} \Delta E / E$ is also small and averaging is weak. Furthermore, in the interval $\cos \theta_{Z}=(0.2-0.5)$ the regeneration factor increases with $\cos \theta_{Z}$. The reason is that in this interval the main term, $\sin ^{2} \Phi_{0}$, starts to "interfere constructively" with the terms produced by the outer shells, $\sin \Phi_{0} \sin \Phi_{i}(i=1,2,3)$, as it has been discussed in section 4.3.

In Fig. 5 and 6 we show dependence of the regeneration factor for the charged current events at SNO. Here we have taken into account the energy resolution of the SNO detector and also performed integration over various energy bins of the observed kinetic energy. On the basis of our analytic formulas and discussion, the interpretation of results of Fig. 5 and 6 is straightforward.

\subsection{Small scale structures: general density profile}

There are small scale structures in the outer mantle of the Earth of depth $(\sim 10) \mathrm{km}$ in which matter has quite different densities (e.g., ocean, rock and soil). In contrast to the ideal PREM model, these structures are not isotropically distributed and can be quite complicated.

In section 4.3 we have shown for the ideal PREM model that contributions produced by structures close the surface of the Earth interfere destructively for small $\cos \theta_{Z}$. Furthermore, averaging over the energy doesn't smooth the dependence of these contributions on $\cos \theta_{Z} \operatorname{com}-$ pletely. This produces an uncertainty for the future high statistics solar neutrino experiments (see also comments in [38]) unless the local density distribution is well known [39]. In view of this we will consider general (not spherically symmetric) density profile.

Suppose neutrinos cross $k$ layers of matter. The density jumps occur in the points $x=x_{i}$ $(i=1, \cdots, k-1) ; x=x_{0}$ and $x=x_{k}$ are the points where neutrinos enter and leave the matter 


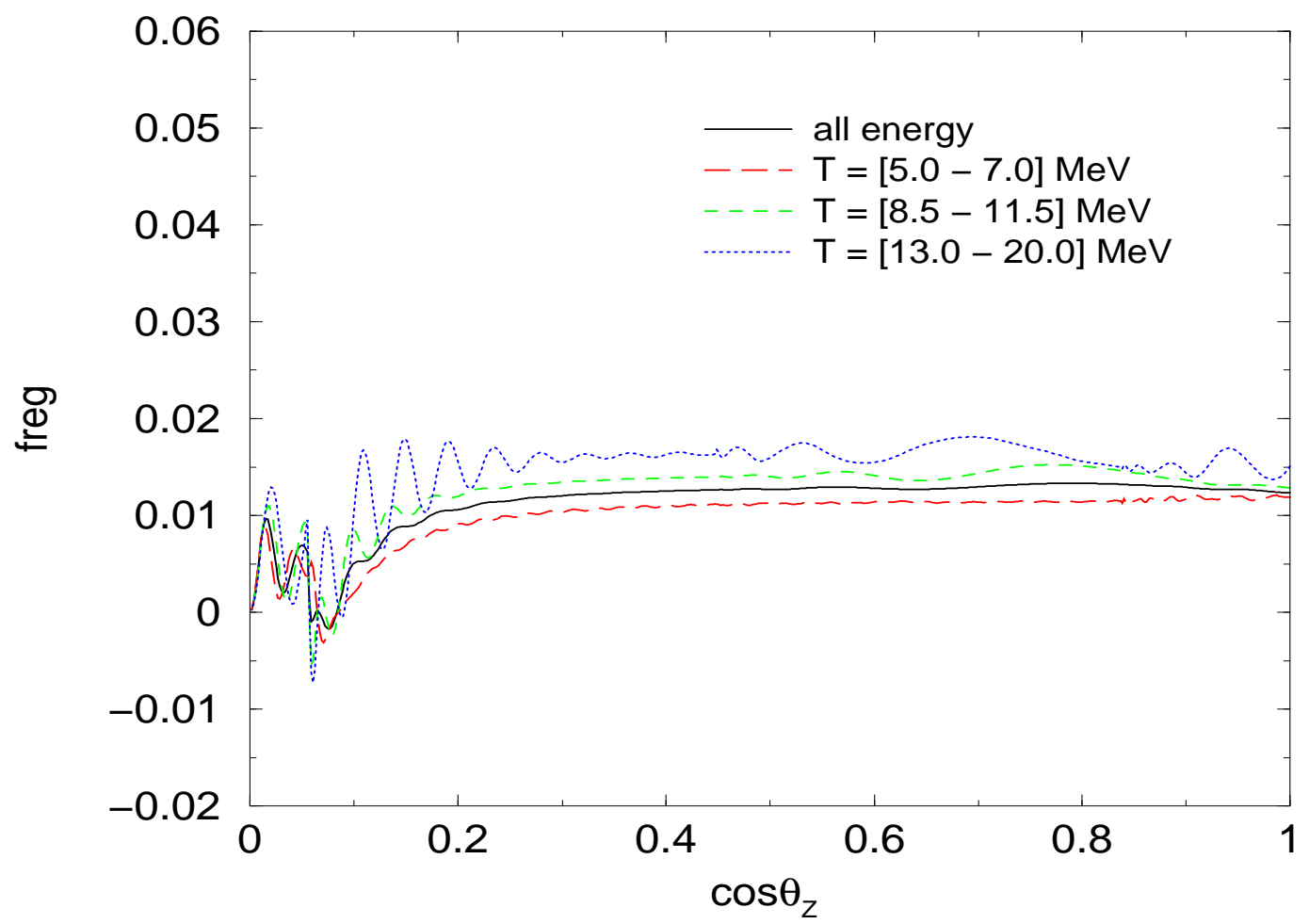

Figure 5: The regeneration factor for the SNO charged current events integrated over different intervals of the observed kinetic energy as function of the zenith angle. We take $\Delta m^{2}=6.3 \times 10^{-5} \mathrm{eV}^{2}$, $\tan ^{2} \theta=0.4$.

correspondingly. Similarly to $(60)$ we parameterize $\dot{\theta}_{m}$ as

$$
\dot{\theta}_{m}=\frac{E \sin 2 \theta}{\Delta m^{2}} \sum_{i=1}^{k-1} \Delta V_{i} \delta\left(x-x_{i}\right),
$$

where the jump of potential at the $i$ th border between layers equals

$$
\Delta V_{i}=V\left(x_{i}+\epsilon\right)-V\left(x_{i}-\epsilon\right), \quad i=1, \cdots, k-1 .
$$

$\epsilon$ is the infinitesimally small distance. Noting that the potential is zero for neutrinos before entering the Earth and after leaving the Earth, we define also

$$
\Delta V_{0}=V\left(x_{0}\right), \quad \Delta V_{k}=-V\left(x_{k}\right) .
$$

Plugging the potential jumps into (18) gives

$$
c\left(x_{k}\right)=-\frac{E \sin 2 \theta}{\Delta m^{2}} \sum_{i=1}^{k-1} \Delta V_{i} e^{2 i \phi_{i}},
$$

where

$$
\phi_{i}=\int_{x_{i}}^{x_{k}} d x \frac{\Delta(x)}{4 E}, \quad i=0, \cdots, k
$$




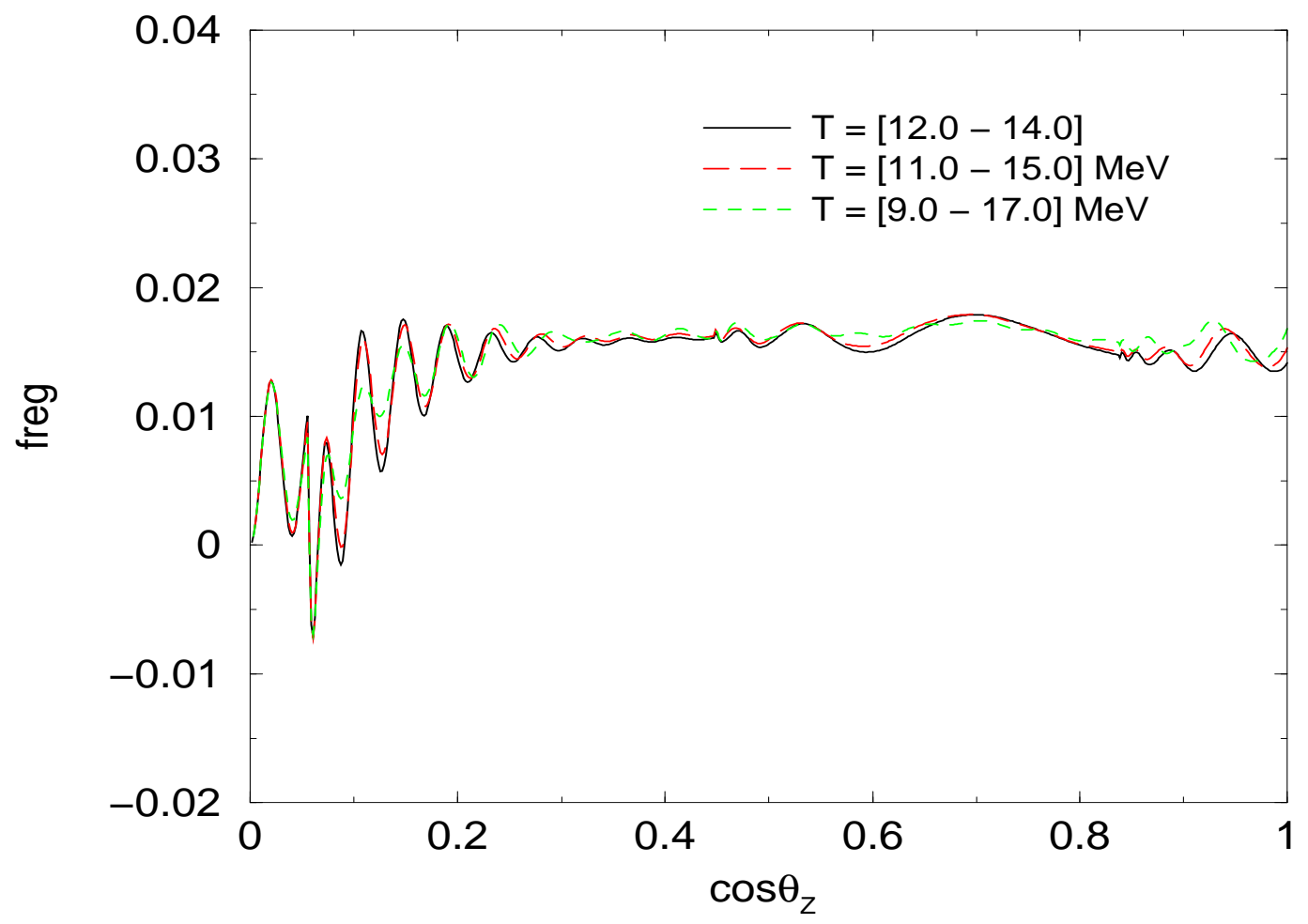

Figure 6: The same as in Fig. 5 for different intervals of averaging and the same middle kinetic energy $T=13 \mathrm{MeV}$.

is the phase acquired from a given border $i$ to the final point of the trajectory (detector).

Now it is straightforward to compute the regeneration factor, and in the leading order in $E V / \Delta m^{2}$ we obtain

$$
\begin{aligned}
f_{r e g} & =\left|\left\langle\nu_{e}\left|U\left(\theta_{m}\left(x_{k}\right)\right) S\left(x_{k}, x_{0}\right) U^{\dagger}\left(\theta_{m}\left(x_{0}\right)\right) U(\theta)\right| \nu_{2}\right\rangle\right|^{2}-\sin ^{2} \theta \\
& =-\frac{E \sin ^{2} 2 \theta}{\Delta m^{2}} \sum_{i=0}^{k} \Delta V_{i} \cos 2 \phi_{i} .
\end{aligned}
$$

In the Appendix $\mathrm{B}$, a direct computation of $f_{\text {reg }}$ for this case is given. Its result coincides with (73) in the leading order in $E V / \Delta m^{2}$. Using this formula one can easily reproduce (63) assuming a symmetric density profile and taking into account that $\Phi_{i}=\left(\phi_{i}-\phi_{k-i}\right)$ for $k=2 n-1$ and $i<n$.

Using (73) it is easy to study averaging effects following the discussion in section 4.4. Structures situated far from the detector have $\phi_{i} \gg 1$. So that, after averaging, remote structures do not produce significant effect. However, if the energy resolution is improved, effects of these remote structures can be large. This agrees with general consideration in [37].

Thus, we arrive at the following conclusion. If $\cos \theta_{Z}$ is large, small scale structures near the entering point can be taken effectively as a single layer. Furthermore, averaging over the energy makes contributions of these small structures to be unimportant.

If $\cos \theta_{Z}$ is small, we can not consider small scale structures near the entering point as a single layer. However uncertainties produced by these structures can be significantly reduced 
if averaging is performed over broad energy interval, i.e. $\Delta E / E \sim 1$. After averaging, the regeneration factor still shows an oscillatory behavior in the region of small $\cos \theta_{Z}$, and this effect is produced by contributions of the shells close to the detector [37].

\section{Conclusion}

We have performed detailed analytic study of the LMA MSW conversion of the solar neutrinos. Our main result is the precise analytic formula for the survival probability which includes nonadiabatic corrections, averaging over the neutrino production region and the Earth regeneration effect. For the $K$ component of the solar neutrino spectrum $(K=p p, p e p, B e, N, O, F, B$, hep) it can be written as

$$
P_{K}=\frac{1}{2}+\frac{1}{2}\left(1-\delta_{K}\right) \cos 2 \theta_{m}\left(\bar{V}_{K}\right) \cos 2 \theta-\left(1-\delta_{K}\right) \cos 2 \theta_{m}\left(\bar{V}_{K}\right) f_{\text {reg }} .
$$

Here the correction due to averaging effect, $\delta_{K}$, is given in Eq. (35); the average values of matter potential in the production regions of $K$ components, $\bar{V}_{K}$, are defined in (30) and their numerical values are presented in the Table 1 . The regeneration factor $f_{\text {reg }}$ is given in (62) for the symmetric density profile and in (73) for general asymmetric density profile.

Effect of averaging over the neutrino production region in the Sun is reduced to specific value of the initial mixing angle in matter which should be taken for the average value of the potential, $\theta_{m}^{0}=\theta_{m}\left(\bar{V}_{K}\right)$, and to the appearance of the correction $\delta_{K}$. We have compared the analytic results with the results of numerical computation and found that maximal deviation $\sim 1.8 \%$ happens for the hep neutrinos. For the boron neutrinos the precision is better than $0.2 \%$.

We have obtained precise analytic formula for the regeneration effect in the Earth using the realistic density profile. We present simple derivation of this formula which uses the adiabatic perturbation theory. Performing also explicit calculations of the evolution in sequent layers we show that this derivation is correct. The analytic formula reproduces results of numerical computations with accuracy determined by $\eta \sim 1-2 \%$.

Essentially the regeneration effect is the sum of contributions from different shells which are determined by jumps of the potential at the borders and by the adiabatic phase acquired inside the outer borders of the corresponding shells. The dependence of regeneration factor on the zenith angle can be understood in terms of interference of contributions from different borders.

The derived analytical formula allows us to understand the effect of averaging over the neutrino energy. Using the analytical formula we have considered effects of small scale structures $(\sim 10 \mathrm{~km})$ of the Earth profile. These effects can be important for small values of $\cos \theta_{Z}$. We stress that local "perturbations" of the density profile can produce sizable uncertainties in $f_{\text {reg }}$.

\section{Acknowledgment}

One of the authors (P.C.H.) would like to thank FAPESP for financial support. A.Y.S. thanks Tokyo Metropolitan University where this work has been accomplished for hospitality. 


\section{Appendix A. Regeneration factor in a symmetric density profile}

Let us derive the expression for the regeneration factor by considering neutrino evolution in sequent layers of the Earth explicitly. First, we find the complete evolution matrix, $\hat{S}$, in the basis of the mass eigenstates $\Psi^{T} \equiv\left(\nu_{1}, \nu_{2}\right)$ :

$$
\Psi\left(x_{f}\right)=\hat{S}\left(x_{f}, x_{0}\right) \Psi\left(x_{0}\right) .
$$

As discussed in section 4.2, the adiabaticity violation effect within layers is suppressed by $2 E /\left(\Delta m^{2} R_{E}\right) \sim 1-2 \%$ in comparison with the leading order Earth matter effect $(\sim \eta)$. Therefore, we neglect the adiabaticity violation within layers.

1). In the case of neutrino propagation in one shell (one layer) we can simply project the adiabatic evolution matrix (11) obtained for the matter eigenstates on to the basis of the mass states. In the leading order in $E V / \Delta m^{2}$ we find

$$
\begin{aligned}
\hat{S} & =\hat{S}_{1}\left(\frac{L}{2},-\frac{L}{2}\right) \\
& =U^{\dagger}(\theta) U\left(\theta_{m R}\right) S^{a d}\left(\frac{L}{2},-\frac{L}{2}\right) U^{\dagger}\left(\theta_{m R}\right) U(\theta)=U\left(\Delta \theta_{m 0}\right) S^{a d}\left(\Phi_{0}\right) U^{\dagger}\left(\Delta \theta_{m 0}\right) \\
& =S^{a d}\left(\frac{L}{2},-\frac{L}{2}\right)+\sin \Delta \theta_{m 0}\left(e^{-i \Phi_{0}}-e^{i \Phi_{0}}\right)\left(\begin{array}{cc}
\sin \Delta \theta_{m 0} & \cos \Delta \theta_{m 0} \\
\cos \Delta \theta_{m 0} & -\sin \Delta \theta_{m 0}
\end{array}\right) \\
& =\left(\begin{array}{cc}
e^{i \Phi_{0}} & 0 \\
0 & e^{-i \Phi_{0}}
\end{array}\right)+\frac{E \Delta V_{0} \sin 2 \theta}{\Delta m^{2}}\left(e^{-i \Phi_{0}}-e^{i \Phi_{0}}\right)\left(\begin{array}{cc}
0 & 1 \\
1 & 0
\end{array}\right) .
\end{aligned}
$$

Here $\Delta \theta_{m 0} \equiv \theta_{m R}-\theta$ is the jump of the mixing angle at the surface of the Earth. We have used Eq. (50), and $\Phi_{0}$ is defined in Eq. (46).

2 ). In the case of two shells crossing, the neutrino encounters three layers (the outer shell is crossed twice). The evolution matrix can be similarly obtained by using the adiabatic evolution

matrix (11) in each layer and by rotation from the matter eigenstates basis in the layer before the border to the basis after the border. As a result, we find

$$
\hat{S}=U\left(\Delta \theta_{m 0}\right) S^{a d}\left(\frac{L}{2}, \frac{L_{1}}{2}\right) S_{1}\left(\frac{L_{1}}{2},-\frac{L_{1}}{2}\right) S^{a d}\left(-\frac{L_{1}}{2},-\frac{L}{2}\right) U^{\dagger}\left(\Delta \theta_{m 0}\right),
$$

where $S_{1}$ is the evolution matrix in the inner shell which has a form similar to Eq. (76) and it can be written as

$$
\begin{aligned}
S_{1} & =U\left(\Delta \theta_{m 1}\right) S^{a d}\left(\frac{L_{1}}{2},-\frac{L_{1}}{2}\right) U^{\dagger}\left(\Delta \theta_{m 1}\right) \\
& =S^{a d}\left(\frac{L_{1}}{2},-\frac{L_{1}}{2}\right)+\frac{E \Delta V_{1}}{\Delta m^{2}} \sin 2 \theta\left(e^{-i \Phi_{1}}-e^{i \Phi_{1}}\right)\left(\begin{array}{ll}
0 & 1 \\
1 & 0
\end{array}\right) .
\end{aligned}
$$

Here $\Delta \theta_{m 1}$ is the jump of mixing angle on the border between the first and the second shells, and $\Phi_{1}$ is defined in Eq. (46). 
Combining the last two formulas we find to the order $E V / \Delta m^{2}$

$$
\begin{aligned}
\hat{S} & =\hat{S}_{2}\left(\frac{L}{2},-\frac{L}{2}\right) \\
& =S^{a d}\left(\frac{L}{2},-\frac{L}{2}\right)+\sum_{i=0}^{1} \frac{E \Delta V_{i} \sin 2 \theta}{\Delta m^{2}}\left(e^{-i \Phi_{i}}-e^{i \Phi_{i}}\right)\left(\begin{array}{ll}
0 & 1 \\
1 & 0
\end{array}\right) .
\end{aligned}
$$

3). Suppose the evolution matrix for $j$ shells crossing ( $2 j-1$ layers) equals

$$
\begin{aligned}
\hat{S} & =\hat{S}_{j}\left(\frac{L}{2},-\frac{L}{2}\right) \\
& =S^{a d}\left(\frac{L}{2},-\frac{L}{2}\right)+\sum_{i=0}^{j-1} \frac{E \Delta V_{i} \sin 2 \theta}{\Delta m^{2}}\left(e^{-i \Phi_{i}}-e^{i \Phi_{i}}\right)\left(\begin{array}{cc}
0 & 1 \\
1 & 0
\end{array}\right) .
\end{aligned}
$$

Consider now the trajectory with $j+1$ shells crossings. The evolution matrix is

$$
\begin{aligned}
\hat{S} & =\hat{S}_{j+1} \\
& =\prod_{i=0}^{j-1}\left[U\left(\Delta \theta_{m i}\right) S^{a d}\left(\frac{L_{i}}{2}, \frac{L_{i+1}}{2}\right)\right] S_{j}\left(\frac{L_{j}}{2},-\frac{L_{j}}{2}\right) \prod_{i=j-1}^{0}\left[S^{a d}\left(-\frac{L_{i+1}}{2},-\frac{L_{i}}{2}\right) U^{\dagger}\left(\Delta \theta_{m i}\right)\right],
\end{aligned}
$$

where $\Delta \theta_{m i}$ defined in (48), is the jump of the mixing angle in matter at the border $R_{i} . S_{j}$ is the evolution matrix in the central shell which can be written similarly to Eq. (77) as

$$
S_{j}=S^{a d}\left(\frac{L_{j}}{2},-\frac{L_{j}}{2}\right)+\frac{E \Delta V_{j}}{\Delta m^{2}} \sin 2 \theta\left(e^{-i \Phi_{j}}-e^{i \Phi_{j}}\right)\left(\begin{array}{ll}
0 & 1 \\
1 & 0
\end{array}\right) .
$$

$\Phi_{j}$ is given in Eq. (46). After insertion into (80), the first term of (81) leads to $\hat{S}_{j}$. The second term in (81) is already of the order $E V / \Delta m^{2}$. Note that $\Delta \theta_{m i}$ is small, as is shown in (50). So, we can approximate $U\left(\Delta \theta_{m i}\right)$ by the unit matrix when plugging the second term in (81) into (80). As a result, we find

$$
\hat{S}=\hat{S}_{j}+\frac{E \Delta V_{j} \sin 2 \theta}{\Delta m^{2}}\left(e^{-i \Phi_{j}}-e^{i \Phi_{j}}\right)\left(\begin{array}{ll}
0 & 1 \\
1 & 0
\end{array}\right) .
$$

Using then expression (79) for $\hat{S}_{j}$, the formula (79) is immediately extended to the case of crossing $j+1$ shells, thus accomplishing the proof. The result for the case of $n$ shells crossing is

$$
\hat{S}=S^{a d}\left(\frac{L}{2},-\frac{L}{2}\right)+\sum_{i=0}^{n-1} \frac{E \Delta V_{i} \sin 2 \theta}{\Delta m^{2}}\left(e^{-i \Phi_{i}}-e^{i \Phi_{i}}\right)\left(\begin{array}{cc}
0 & 1 \\
1 & 0
\end{array}\right) .
$$

Using (83) we obtain the expression for the regeneration factor in the leading order in $E V / \Delta m^{2}$ as

$$
\begin{aligned}
f_{\text {reg }} & =\left|\sin \theta e^{-i \Phi_{0}}+\cos \theta \sum_{i=0}^{n-1} \frac{E \Delta V_{i} \sin 2 \theta}{\Delta m^{2}}\left(e^{-i \Phi_{i}}-e^{i \Phi_{i}}\right)\right|^{2}-\sin ^{2} \theta \\
& =\frac{2 E \sin ^{2} 2 \theta}{\Delta m^{2}} \sin \Phi_{0} \sum_{i=0}^{n-1} \Delta V_{i} \sin \Phi_{i},
\end{aligned}
$$

where $\Phi_{i}$ is given in (46) and $\Delta V_{i}$ is defined in (47). This expression coincides with (62) or (63) which have been obtained in section 4.3 using the adiabatic perturbation theory. 


\section{Appendix B. Regeneration in asymmetric density profile}

As in the section 4.5, we define

$$
\Delta \theta_{i} \equiv \theta_{m}\left(x_{i}+\epsilon\right)-\theta_{m}\left(x_{i}-\epsilon\right), \quad i=0, \cdots, k,
$$

where $\Delta \theta_{0}=\theta_{m}\left(x_{0}\right)-\theta$ and $\Delta \theta_{k}=\theta-\theta_{m}\left(x_{k}\right)$, and $x=x_{i}$ are the points of density jumps. $x_{0}$ and $x_{k}$ are the initial and final points of neutrino trajectory in matter. We will use the following expression:

$$
\sin \Delta \theta_{i} \approx \frac{E \Delta V_{i}}{\Delta m^{2}} \sin 2 \theta, \quad \cos \Delta \theta_{i} \approx 1
$$

which is a good approximation in the leading order in $E \Delta V / \Delta m^{2} . \Delta V_{i}$ is given in (69). Neglecting the adiabaticity violation within each layer, we obtain the evolution matrix $\hat{S}$ as

$$
\hat{S}=\left(\prod_{i=k}^{1} U^{\dagger}\left(\Delta \theta_{i}\right) S^{a d}\left(x_{i}, x_{i-1}\right)\right) U^{\dagger}\left(\Delta \theta_{0}\right) .
$$

$S^{a d}\left(x, x_{0}\right)=S^{a d}(\phi(x))$ is the adiabatic evolution matrix given in (11).

We approximate $U^{\dagger}\left(\Delta \theta_{i}\right)$ as

$$
U^{\dagger}\left(\Delta \theta_{i}\right)=\left(\begin{array}{ll}
1 & 0 \\
0 & 1
\end{array}\right)+Q\left(\Delta \theta_{i}\right)
$$

where

$$
Q\left(\Delta \theta_{i}\right) \equiv\left(\begin{array}{cc}
0 & -\sin \Delta \theta_{i} \\
\sin \Delta \theta_{i} & 0
\end{array}\right)
$$

Straightforward computation gives the following result in the leading order in $E V / \Delta m^{2}$

$$
\begin{aligned}
\hat{S} & =S^{a d}\left(x_{k}, x_{0}\right)+Q\left(\Delta \theta_{k}\right) S^{a d}\left(x_{k}, x_{0}\right)+S^{a d}\left(x_{k}, x_{0}\right) Q\left(\Delta \theta_{0}\right) \\
& +\sum_{i=1}^{k-1} S^{a d}\left(x_{k}, x_{i}\right) Q\left(\Delta \theta_{i}\right) S^{a d}\left(x_{i}, x_{0}\right) \\
& =S^{a d}\left(x_{k}, x_{0}\right)+\sum_{i=0}^{k}\left(\begin{array}{cc}
0 & -\sin \Delta \theta_{i} e^{i\left(2 \phi_{i}-\phi_{0}\right)} \\
\sin \Delta \theta_{i} e^{-i\left(2 \phi_{i}-\phi_{0}\right)} & 0
\end{array}\right) .
\end{aligned}
$$

$\phi_{i}$ is defined in (72). Then, using (86), the regeneration factor can be directly computed in the first order in $E V / \Delta m^{2}$ as

$$
\begin{aligned}
f_{r e g} & =\left|\sin \theta e^{-i \phi_{0}}-\cos \theta \sum_{i=0}^{k} \sin \Delta \theta_{i} e^{i\left(2 \phi_{i}-\phi_{0}\right)}\right|^{2}-\sin ^{2} \theta \\
& =-\frac{E \sin ^{2} 2 \theta}{\Delta m^{2}} \sum_{i=0}^{k} \Delta V_{i} \cos 2 \phi_{i} .
\end{aligned}
$$

It coincides with (73). 


\section{Note added}

This note has been added on request of the referee.

1). After the present paper had appeared in the hep-ph archive [hep-ph/0404042], the preprint by Akhmedov et. al., [hep-ph/0404083], has been published in which the analytic integral formula is given for the the regeneration effect in the Earth in the three neutrino framework. In the first version of [hep-ph/0404083] the correct oscillation phase in this integral formula has been introduced on the "heuristic" basis: it does not follow from their perturbation theory. Correct integral formula (with the correct phase) has been derived for the first time in the paper by Ioannisian and Smirnov, [hep-ph/0404060]. Later in the Journal version JHEP 0405 (2004) 057, Akhmedov et. al., have also presented derivation of correct phase.

Let us now compare the results of papers [hep-ph/0404060], [hep-ph/0404083] with the results of present paper. (We will use the Akhmedov's et. al. results in the limit of zero 1-3 mixing.)

In Ioannisian and Smirnov paper hep-ph/0404060 and Akhmedov et. al. paper JHEP 0405 (2004) 057 the integral formula has been obtained using the improved perturbation theory in the small parameter $\eta \equiv 2 E V(x) / \Delta m^{2}$. In the present paper we use the adiabatic perturbation theory. It can be shown that in the lowest order in $\eta$ both approaches coincide. Indeed, inserting expression for $\dot{\theta}_{m}$ from (7) into (18) and performing integration by parts in Eq. (18) of the present paper one can derive the integral formula.

This formula is valid for arbitrary density profile provided that the condition $\eta \ll 1$ is satisfied. Inserting the potential of PREM model (Eqs. $(47,53))$ into the integral formula one can reproduce the result (63). However technically the use of formula (18) of the present paper is more convenient for the derivation of (63) since the derivative $d \theta_{m} / d x$ gives $\delta$ - functions at the borders of layers and further integration becomes trivial.

2). The KamLAND collaboration has published recently results of improved measurements of oscillations on the basis of 766.3 ton-year exposure [T. Araki et. al., hep-ex/0406035]. In assumption of the CPT conservation, the global analysis of the solar neutrino data and KamLAND gives slightly $(\sim 10 \%)$ higher best fit value $\Delta m^{2}=8 \cdot 10^{-5} \mathrm{eV}^{2}$ than it was before. The increase of $\Delta m^{2}$ leads to the corresponding small decrease of the adiabaticity parameter $\gamma$ and the expansion parameter $\eta$ for a given energy of neutrinos. Therefore increase in $\Delta m^{2}$ (i) further improves the adiabatic perturbation theory and implies that the non-adiabatic correction for probability in the Sun is smaller; (ii) diminishes the Earth matter regeneration effect; (iii) makes our analytic formula for $f_{\text {reg }}$ preciser. Notice that the analytic study of this paper has a general character and does not rely on particular values of $\Delta m^{2}$. We use specific value of $\Delta m^{2}$ for illustration only.

\section{References}

[1] L. Wolfenstein, Phys. Rev. D 17, 2369 (1978); L. Wolfenstein, in "Neutrino-78", Purdue Univ. C3 - C6, (1978).

[2] S. P. Mikheyev and A. Yu. Smirnov, Yad. Fiz. 42, 1441 (1985) [ Sov. J. Nucl. Phys. 42, 913 (1985)]; Nuovo Cim. C9, 17 (1986); S. P. Mikheyev and A. Yu. Smirnov, ZHETF, 91, (1986), [Sov. Phys. JETP, 64, 4 (1986)] (reprinted in "Solar neutrinos: the first thirty years", Eds. J.N.Bahcall et. al.). 
[3] Q. R. Ahmad et al., SNO collaboration Phys. Rev. Lett 87(2001)071301; ibidem 89(2002)011301; ibidem 89(2002)011302.

[4] Q. R. Ahmad et al., SNO collaboration, nucl-ex/0309004.

[5] Super-Kamiokande collaboration, S. Fukuda et al., Phys. Rev. Lett. 86(2001)5651; Phys. Rev. Lett. 86(2001) 5656; Phys. Lett. B 539(2002)179.

[6] M. B. Smy et al., Super-Kamiokande Collaboration, Phys. Rev. D69(2004)011104.

[7] K. Eguchi et al., KamLAND Coll., Phys. Rev. Lett. 90(2003)021802[hep-ex/0212021].

[8] A. B. Balantekin and H. Yüksel, Phys. Rev. D68(2003)113002[hep-ph/0309079].

[9] G.L. Fogli, E. Lisi, A. Marrone, A. Palazzo, Phys. Lett. B583(2004)149[hep-ph/0309100].

[10] M. Maltoni, T. Schwetz, M. A. Tortola, J.W.F. Valle, Phys. Rev. D68(2003)113010[hep$\mathrm{ph} / 0309130]$.

[11] P. Aliani, V. Antonelli, M. Picariello, E. Torrente-Lujan, hep-ph/0309156.

[12] P. Creminelli, G. Signorelli, A. Strumia, hep-ph/0102234, v.5, Sept. 15 (2003).

[13] A. Bandyopadhyay, S. Choubey, S. Goswami, S. T. Petcov, D.P. Roy, Phys. Lett. B583(2004)134[hep-ph/0309174].

[14] P. C. de Holanda, A.Yu. Smirnov, JCAP 0302(2003)001 [hep-ph/0212270].

[15] P. C. de Holanda, A.Yu. Smirnov, hep-ph/0309299, to be published in Astropart. Phys..

[16] J. N. Bahcall, C. Pena-Garay, JHEP 0311(2003)004, and references therein.

[17] UNO Proto-collaboration, UNO Whitepaper: Physics Potential and Feasibility of UNO, SBHEP-01-03(2000), http://nngroup.physics.sunysb.edu/uno/; see also C. K. Jung 2002, hep-ex/0005046

[18] Next-Generation Cherenkov Detector Hyper-Kamiokande, $\quad$ K. Nakamura, talk at Neutrinos and Implications for Physics Beyond the Standard Model 2002, http://insti.physics.sunysb.edu/itp/conf/neutrino/talks/nakamura.pdf.

[19] A European Megaton Project at Fréjus, L. Mosca, talk at TAUP 2003, http://mocha.phys.washington.edu/ int_talk/WorkShops/TAUP03/.

[20] A. Messiah, in Proceedings of the 6th Moriond Workshop On Massive Neutrino in Particle Physics and Astrophysics, ed. O. Fackler and J. Tran Thanh Van, 1986.

[21] S. J. Parke, Phys. Rev. Lett. 57(1986)1275.

[22] S. P. Mikheev and A. Yu. Smirnov, Sov. Phys. JETP 65(1987)230.

[23] L. Landau, Phys. Z. Sov2(1932)46; C. Zener, Proc. Roy. Soc. Lon. A137(1932)696. 
[24] A. Friedland, hep-ph/0106042, contributed to 2nd Frontiers in Contemporary Physics: The Inner Space Outer Space Connection, Nashville, Tennessee, 2001.

[25] S.T. Petcov, Phys. Lett. B200(1988)373; P.I. Krastev and S.T. Petcov, Phys. Lett. B207(1998)64.

[26] John N. Bahcall, M. H. Pinsonneault, Sarbani Basu, Astrophys. J. 555(2001)990[astro$\mathrm{ph} / 0010346]$

[27] S. P. Mikheyev and A. Yu. Smirnov, '86 Massive Neutrinos in Astrophysics and in Particle Physics, proceedings of the Sixth Moriond Workshop, edited by O. Fackler and J. Trân Thanh Vân (Editions Frontières, Gif-sur-Yvette, 1986), pp. 355.

[28] J. Bouchez et. al., Z. Phys. C32, 499 (1986); M. Cribier et. al., Phys. Lett. B 182, 89 (1986); E. D. Carlson, Phys. Rev. D34, 1454 (1986) .

[29] A.J. Baltz and J. Weneser, Phys. Rev. D35, 528 (1987); A. Dar et. al., Phys. Rev. D 35 (1987) 3607; S. P. Mikheyev and A. Yu. Smirnov, Sov. Phys. Usp. 30 (1987) 759-790; L. Cherry and K. Lande, Phys. Rev D 363571 (1987); S. Hiroi, H. Sakuma, T. Yanagida, M. Yoshimura, Phys. Lett. B198 403, (1987) and Prog. Theor. Phys. 78 1428, (1987); A. J. Baltz and J. Weneser, Phys. Rev. D37, 3364 (1988). M. Spiro and D. Vignaud, Phys. Lett. B 242297 (1990).

[30] A. J. Baltz and J. Weneser, Phys. Rev. D50, 5971 (1994); ibid D51, 3960 (1994); M. Maris and S.T. Petcov, Phys. Rev. D62(2000)093006; N. Hata and P. Langacker, Phys. Rev. D 48(1993)2937 and Phys. Rev. D 50(1994)632; E. Lisi, D. Montanino, Phys. Rev. D56(1997)1792; J. M. Gelb, Wai-kwok Kwong, S. P. Rosen, Phys. Rev. Lett. 78(1997)2296.

[31] Q. Y. Liu, M. Maris and S. T. Petcov Phys. Rev. D 565991 (1997); M. Maris, S.T. Petcov, Phys. Rev. D 567444 ,1997. M. Narayan, G. Rajasekaran, R. Sinha; Mod. Phys. Lett. A13: 1915 (1998); A. de Gouvea, A. Friedland, H. Murayama, hep-ph/9910286; G.L. Fogli, E. Lisi, D. Montanino, Phys. Rev. D 61073009 (2000); G.L. Fogli, E. Lisi, D. Montanino, A. Palazzo, hep-ph/0008012.

[32] J. N. Bahcall, P. I. Krastev, A. Yu. Smirnov, Phys. Rev. D60(1999) 093001;P. C. de Holanda, C. Pẽna-Garay, M.C. Gonzalez-Garcia, J. W. F. Valle, Phys. Rev. D60(1999)093010; A. H. Guth, L. Randall, M. Serna, JHEP 9908(1999)018.

[33] V. K. Ermilova, V. A. Tsarev, and V. A. Chechin, Short Notices Lebedev Inst. 5, 26 (1986); E.Kh. Akhmedov, Yad. Fiz. 47, 475 (1988).

[34] P. I. Krastev and A. Yu. Smirnov, Phys. Lett. B 226(1989)341; J.M. Losecco, Phys. Rev. D47(1993)2032; Q. Y. Liu and A. Yu. Smirnov, Nucl. Phys. B 524(1998)505; Q. Y. Liu, S. P. Mikheyev, and A. Yu. Smirnov, Phys. Lett. B440(1998)319; S.T. Petcov, Phys. Lett. B 434(1998)321; Kh. Akhmedov, A. Dighe, P. Lipari, and A. Yu. Smirnov, Nucl. Phys. B542(1999)3; E. Kh. Akhmedov, hep-ph/9903302; E.K. Akhmedov, Nucl. Phys. B538(1999)25; M.V. Chizhov, S.T. Petcov, Phys. Rev. Lett. 83(1999)1096; E.K. Akhmedov, A.Yu. Smirnov, Phys. Rev. Lett. 85(2000)3978.

[35] A.M. Dziewonski and D.L. Anderson, Phys. Earth. Planet. Inter.25(1981)297. 
[36] E. Lisi and D. Montanino, Phys. Rev. D56(1997)1792.

[37] A. Ioannissian, A. Smirnov, hep-ph/0404060.

[38] G. L. Fogli, E. Lisi, A. Marrone, D. Montanino, A. Palazzo, Phys. Rev. D66(2002)053010.

[39] A. Ioannissian, A. Smirnov, in preparation. 